\title{
Breaking Instance-Independent Symmetries in Exact Graph Coloring
}

\author{
Arathi Ramani \\ Igor L. Markov \\ Karem A. Sakallah \\ Department of Electrical Engineering and Computer Science \\ University of Michigan, Ann Arbor, USA
}

Fadi A. Aloul

Department of Computer Engineering

American University in Sharjah, UAE

\author{
RAMANIA@UMICH.EDU \\ IMARKOV@EECS.UMICH.EDU \\ KAREM@EECS.UMICH.EDU
}

FALOUL@UMICH.EDU

\begin{abstract}
Code optimization and high level synthesis can be posed as constraint satisfaction and optimization problems, such as graph coloring used in register allocation. Graph coloring is also used to model more traditional CSPs relevant to AI, such as planning, time-tabling and scheduling. Provably optimal solutions may be desirable for commercial and defense applications. Additionally, for applications such as register allocation and code optimization, naturally-occurring instances of graph coloring are often small and can be solved optimally. A recent wave of improvements in algorithms for Boolean satisfiability (SAT) and 0-1 Integer Linear Programming (ILP) suggests generic problem-reduction methods, rather than problem-specific heuristics, because (1) heuristics may be upset by new constraints, (2) heuristics tend to ignore structure, and (3) many relevant problems are provably inapproximable.

Problem reductions often lead to highly symmetric SAT instances, and symmetries are known to slow down SAT solvers. In this work, we compare several avenues for symmetry breaking, in particular when certain kinds of symmetry are present in all generated instances. Our focus on reducing CSPs to SAT allows us to leverage recent dramatic improvement in SAT solvers and automatically benefit from future progress. We can use a variety of black-box SAT solvers without modifying their source code because our symmetry-breaking techniques are static, i.e., we detect symmetries and add symmetry breaking predicates (SBPs) during pre-processing.

An important result of our work is that among the types of instance-independent SBPs we studied and their combinations, the simplest and least complete constructions are the most effective. Our experiments also clearly indicate that instance-independent symmetries should mostly be processed together with instance-specific symmetries rather than at the specification level, contrary to what has been suggested in the literature.
\end{abstract}

\section{Introduction}

Detecting and using problem structure, such as symmetries, can often be very useful in accelerating the search for solutions of constraint satisfaction problems (CSPs). This is particularly true for algorithms which perform exhaustive searches and benefit from pruning the search tree. This work conducts a theoretical and empirical study of the impact of breaking structural symmetries in 0-1 ILP reductions of the exact graph coloring problem 
which has applications in a number of fields. For example, in compiler design, many techniques for code optimization and high-level synthesis operate with relatively few objects at a time. Graph coloring used for register allocation during program compilation (Chaitin, Auslander, Chandra, Cocke, Hopkins, \& Markstein, 1981) is limited by small numbers of registers in embedded processors as well as by the number of local variables and virtual registers. Graph coloring is also relevant to AI applications such as planning, scheduling, and map coloring. Recent work on graph coloring in AI has included algorithms based on neural networks (Jagota, 1996), evolutionary algorithms (Galinier \& Hao, 1999), scatter search (J.-P. Hamiez, 2001) and several other approaches discussed in Section 2. While many of these search procedures are heuristic, our work focuses on exact graph coloring, which is closely related to several useful combinatorial problems such as maximal independent set and vertex cover. We seek provably optimal solutions because they may be desirable in commercial and defense applications for competitive reasons, and can often be found. Our work focuses on solving exact graph coloring by reduction to 0-1 ILP. While the idea of solving $\mathcal{N} \mathcal{P}$ - complete problems by reduction is well-known, it is rarely used in practice because algorithms developed for "standard" problems, such as SAT, may not be competitive with domain-specific techniques that are aware of problem structure. However, many applications imply problem-specific constraints and non-trivial objective functions. These extensions may upset heuristics for standard problems. Heuristics, particularly those based on local search, often fail to use structure in problem instances (Prestwich, 2002) and are inefficient when used with problem reductions. In contrast, exact solvers based on branch-and-bound and back-tracking tend to adapt to new constraints and can be applied through problem reduction. There is a growing literature on handling structure in optimal solvers (Aloul, Ramani, Markov, \& Sakallah, 2003; Crawford, Ginsberg, Luks, \& Roy, 1996; Huang \& Darwiche, 2003), and our work falls into this category as well.

The NP-spec project (Cadoli, Palopoli, Schaerf, \& Vasileet, 1999) offers a framework for formulating a wide range of combinatorial problems and automatically reducing their instances to instances of Boolean satisfiability. This approach is attractive because it circumvents problem-specific solvers and leverages recent breakthroughs in Boolean satisfiability (Moskewicz, Madigan, Zhao, Zhang, \& Malik, 2001). However, this approach remains unexplored in practice, possibly because the efficiency of problem-solving may be reduced when domain-specific structure is lost during problem reductions. This drawback is addressed by recent work on the detection of structure, particularly symmetry, in SAT and 0-1 ILP instances in order to accelerate exact solvers (Crawford et al., 1996; Aloul et al., 2003; Aloul, Ramani, Markov, \& Sakallah, 2004). In these papers, symmetries in a SAT/0-1 ILP instance are detected by reduction to graph automorphism, i.e. the formula is represented by a graph and the automorphism problem for that graph is solved using graph automorphism software packages (McKay, 1990; Darga, Liffiton, Sakallah, \& Markov, 2004). Until recently, this type of symmetry detection was frequently inefficient because solving the automorphism problem for large graphs can be time-consuming. However, more recent automorphism software (Darga et al., 2004) has removed this bottleneck to a large extent. Moreover, adding simple symmetry breaking predicates as new constraints significantly speeds up exact SAT solvers (Aloul et al., 2003). This work can be viewed as a case study of symmetry breaking in problem reductions, as we focus on graph coloring and its variants that can be reduced to Boolean satisfiability and 0-1 ILP. Our main goals are to (i) 
accelerate optimal solving of graph coloring instances, and (ii) compare different strategies for breaking instance-independent symmetries. There are two distinct sources of symmetries in graph-coloring instances: (i) colors can be arbitrarily permuted (instance-independent symmetries), and (ii) some graphs may be invariant under certain permutations of vertices (instance-dependent symmetries). Previous work (Crawford et al., 1996; Aloul et al., 2003, 2004) deals only with instance-dependent symmetries in SAT and 0-1 ILP instances. Symmetries are first detected by reduction to graph automorphism and then broken by adding symmetry breaking predicates (SBPs) to the formulation. The advantage of such a strategy is that every instance-independent symmetry is also instance-dependent, whereas the reverse does not hold. Symmetries that exist due to problem formulation appear in every instance of the problem, in addition to symmetries that exist due to specific parameter values for an instance. Given that there may be many instance-specific symmetries, one may process all symmetries at once using publicly available symmetry processing packages such as Shatter (Aloul et al., 2003; Aloul, Markov, \& Sakallah, 2003). Alternatively, one may add symmetry breaking predicates for instance-independent symmetries early, hoping to speed-up the processing of remaining symmetries. This type of symmetry breaking has not been discussed in earlier work (Aloul et al., 2003, 2003), and in this paper we study its utility for the graph coloring problem.

Our work deals with symmetries of problem and instance descriptions; we distinguish (i) symmetries of generic problem specifications from (ii) symmetries of problem-instance data. The former symmetries translate to the latter but not the other way around an example from graph coloring is given by color permutations versus automorphisms of specific graphs. While both types of symmetries can be detected by solving the graph automorphism problem, symmetries in specifications can often be captured manually, whereas capturing symmetries in problem instances may require large-scale computation and nontrivial software. Indeed, when specification-level symmetries are instantiated, the size of their support (the number of objects moved) typically increases dramatically. For example, color permutations in graph coloring should be simultaneously applied to every vertex of a graph in question. Detecting symmetries with larger support seems like a waste of computational effort. To this end, recent work on breaking symmetries in specifications (Cadoli \& Mancini, 2003) prefers instance-independent techniques and breaks symmetries only at the specification level. This approach is particularly relevant with constraint solvers and languages that process problem specifications prior to seeing actual problem instances and can amortize the symmetry-detection effort. Also, in a more general setting, using instanceindependent symmetry breaking does not rule out applying redundant (or complementary) instance-specific techniques at a later stage.

Until recently automatic symmetry detection had been a serious bottleneck in handling symmetries. For example, if graph automorphism is solved using the program NAUTY (McKay, 1990), detecting symmetries often can take longer than constraint solving without symmetry breaking. This was observed for microprocessor verification SAT instances by Aloul et. al. in 2002 (Aloul et al., 2003). Therefore, detecting symmetries early and representing them in a more structured way appears attractive, especially given that this may potentially increase the efficiency of symmetry-breaking. However, the symmetrydetection bottleneck has recently been eliminated in many applications with the software tool SAUCY (Darga et al., 2004) that often finds symmetries of practical graphs many times 
faster than NAUTY. This development undermines, to some extent, the potential benefits of symmetry processing at the specification level and puts the spotlight on symmetry-breaking. To that end, SBPs added in different circumstances may have different efficiency, and while it is unclear a priori which approach is more successful, the differences in performance may be significant. Since SBPs appear to the solver as additional constraints, they may either speed up or frustrate the solver (the latter effect is clearly visible in our experiments with CPLEX). Outcomes of practical experiments are also affected by recent dramatic improvements in the efficiency of symmetry-breaking predicates (Aloul et al., 2003, 2004). While it seems difficult to justify any particular expectation for empirical performance, we are fortunate to observe clear trends in experimental data presented in Section 4 and summarize them with simple rules.

While we focus on graph coloring instances, our techniques are immediately applicable to related CSP problems, e.g., those produced by adding new types of constraints that can be easily expressed in SAT or 0-1 ILP when graph coloring is converted to those generic problems. We also expect that our conclusions about symmetry-breaking carry over to other CSPs that can be economically reduced to SAT and 0-1 ILP, e.g., maximum independent set, minimum dominating set, etc. Another advantage of our approach is being able to use a variety of existing and future SAT and 0-1 ILP solvers without modifying their source code. Unfortunately, this precludes the use of dynamic symmetry-breaking that would require modifying the source code and may adversely affect performance by disturbing the fragile balance between the amount of reasoning and searching performed by modern SAT solvers. Specifically, heuristics for variable ordering and decision selection may be affected, as well as the recording of learned conflict clauses (nogoods).

The main contributions of this work are listed below.

- Using the symmetry breaking flow for pseudo-Boolean (PB) formulas described by Aloul et. al in 2004 (Aloul et al., 2004), we detect and break symmetries in DIMACS graph coloring benchmarks expressed as instances of 0-1 ILP. We show that instancedependent symmetry breaking enables many medium-sized instances to be optimally solved in reasonable time on commodity PCs

- We propose instance-independent techniques for breaking symmetries during problem formulation, assess their relative strength and completeness, and evaluate them empirically using well-known academic and commercial tools

- We show empirically that instance-dependent techniques are, in general, more effective than instance-independent symmetry breaking for the benchmarks in question. In fact, only the simplest and least complex instance-independent SBPs are competitive

The remaining part of the paper is organized as follows. Section 2 covers background on graph coloring, SAT and 0-1 ILP, as well as previous work on symmetry breaking. Instanceindependent symmetry breaking predicates are discussed in Section 3. Section 4 presents our empirical results and Section 5 concludes the paper. The Appendix gives detailed results for the queens family of instances. 


\section{Background and Previous Work}

This section discusses problem definitions and applications of some existing algorithms for exact graph coloring. We also discuss previous work on symmetry breaking for SAT and 0-1 ILP in some detail.

\subsection{Graph Coloring}

Given an undirected graph $G(V, E)$, a vertex coloring of the graph is an assignment of a label (color) to each node such that the labels on adjacent nodes are different. A minimum coloring uses the smallest possible number of colors, known as the chromatic number of a graph. The decision version of graph coloring $(K$-coloring) asks whether vertices in a graph can be colored using $\leq K$ colors for a given $K$.

A clique of an undirected graph $G(V, E)$ is a set of mutually adjacent vertices in the graph. The maximum clique problem consists of seeking a clique of maximal size, i.e., a clique with at least as many vertices as any other clique in the graph. The maximum clique and graph coloring problems are closely related. Specifically, the max-clique size is a lower bound on the chromatic number of the graph. Over the years, a number of different algorithms for solving graph coloring have been developed, because of its fundamental importance in computer science. These algorithms fall into three broad categories: polynomial-time approximation schemes, optimal algorithms, and heuristics. We briefly discuss work in each of these categories below. There are a number of online resources on graph coloring (Trick, 1996; Culberson, 2004) that offer more detailed bibliographies.

As far as approximation schemes are concerned, the most common technique used is successive augmentation. In this approach a partial coloring is found on a small number of vertices and this is extended vertex by vertex until the entire graph is colored. Examples include the algorithms by Leighton (Leighton, 1979) for large scheduling problems, and by Welsh and Powell (Welsh \& Powell, 1967) for time-tabling. More recent work has attempted to tighten the worst-case bounds on the chromatic number of the graph. The algorithm providing the currently best worst-case ratio (number of colors used divided by optimal number) is due to Haldorsson (Haldorsson, 1990), and guarantees a ratio of no more than

$O\left(\frac{n(\log \log n)^{2}}{(\log n)^{3}}\right)$, where $n$ is the number of vertices. General heuristic methods that have been tried include simulated annealing (Chams, Hertz, \& Werra, 1987; Aragon, Johnson, McGeoch, \& Schevon, 1991) and tabu search (Hertz \& Werra, 1987). A well-known heuristic that is still widely used is the DSATUR algorithm by Brelaz (Brelaz, 1979) which colors vertices according to their saturation degree. The saturation degree of a vertex is the number of different colors to which it is adjacent. The DSATUR heuristic repeatedly picks a vertex with maximal saturation degree and colors it with the lowest-numbered color possible. This heuristic is optimal for bipartite graphs. Algorithms for finding optimal colorings are frequently based on implicit enumeration, and are discussed in more detail later in this section. Both the graph coloring and max-clique problems are $\mathcal{N} \mathcal{P}$-complete (Garey \& Johnson, 1979) and even finding near-optimal solutions with good approximation guarantees is $\mathcal{N} \mathcal{P}$-hard (Feige, Goldwasser, Lovasz, Safra, \& Szege, 1991). The inapproximability of graph coloring suggests that it may be more difficult to solve heuristically than, say, the Traveling Salesman Problem for which Polynomial-Time Approximation Schemes (PTAS) 
are known for Euclidean and Manhattan graphs. For this and a number of other reasons, we study optimal graph coloring and many application-derived instances that are solvable in reasonable time. Several applications are outlined next.

Time-TABling And Scheduling problems involve placing pairwise restrictions on jobs that cannot be performed simultaneously. For example, two classes taught by the same faculty member cannot be scheduled in the same time slot. The problem has been studied in previous work by Leighton (Leighton, 1979) and De Werra (Werra, 1985). More generally, graph coloring is an important problem in Artificial Intelligence because of its close relationship to planning and scheduling. Several traditional AI techniques have been applied to this problem, including parallel algorithms using neural networks (Jagota, 1996). Genetic and hybrid evolutionary algorithms have also been developed, notably by Galinier et. al. in 1999 (Galinier \& Hao, 1999), in addition to more traditional optimization methodology, such as scatter search (J.-P. Hamiez, 2001). There have also been studies of benchmarking models for graph coloring, such as the recent work by Walsh (Walsh, 2001), which shows that graphs with high vertex degrees are more likely to occur in real-world applications.

Register Allocation is a very active application of graph coloring. This problem seeks to assign variables to a limited number of hardware registers during program execution. Accessing variables in registers is much faster than fetching them from memory. However, the number of registers is limited and is typically much smaller than the number of variables. Therefore, multiple variables must be assigned to the same register. There are restrictions on these assignments. Two variables conflict with each other if they are live at the same time, i.e. one is used both before and after the other within a short period of time (for instance, within a subroutine). The goal is to assign variables that do not conflict so as to minimize the use of non-register memory. To formalize this, one creates a graph where nodes represent variables and edges represent conflicts between variables. A coloring maps to a conflict-free assignment, and if the number of registers exceeds the chromatic number, a conflict-free register assignment exists (Chaitin et al., 1981).

Printed Circuit Board Testing (Garey \& Johnson, 1979) involves the problem of testing printed circuit boards (PCBs) for unintended short circuits (caused by stray lines of solder). This gives rise to a graph coloring problem in which the vertices correspond to the nets on board and there is an edge between two vertices if there is a potential for a short circuit between the corresponding nets. Coloring the graph corresponds to partitioning the nets into "supernets," where the nets in each supernet can be simultaneously tested for shorts against all other nets, thereby speeding up the testing process.

RADIO FREQUENCY ASSIGNMENT for broadcast services in geographic regions (including commercial radio stations, taxi dispatch, police and emergency services). The list of all possible frequencies is fixed by government agencies, but adjacent geographic regions cannot use overlapping frequencies. To reduce frequency assignment to graph coloring, each geographic region needing $\mathrm{K}$ frequencies is represented with a $K$-clique, and all $N \times K$ possible bipartite edges are introduced between two geographically adjacent regions needing $N$ and $K$ frequencies respectively.

Other applications of graph coloring in circuit design and layout include circuit clustering, scheduling for signal flow graphs, and many others. Benchmarks from these applications are not publicly available, and therefore do not appear in this paper. However, all the symmetry breaking techniques described here extend to instances from any application. The 
benchmarks we use here do include register allocation, $n$-queens, and several other applications discussed in more detail in Section 4. Empirically, we observe that many of the instances in this paper can be optimally solved in reasonable time, especially when symmetry breaking is employed. Since this work deals with finding optimal solutions for graph coloring, we discuss previous work on finding exact algorithms for this problem in some detail.

The literature on exact graph coloring includes generic algorithms (Kubale \& Jackowski, 1985) and specialized algorithms for a particular application, such as Chaitin's register allocation algorithm (Chaitin et al., 1981). At the moment, there does not appear to be a comprehensive survey of techniques for this problem. However, online surveys (Trick, 1996; Culberson, 2004) contain reasonably large bibliographies and even downloadable source code for coloring algorithms in some cases. Published algorithms for finding optimal graph colorings are mainly based on implicit enumeration. The algorithm proposed by Brown (Brown, 1972) enumerates solutions for a given instance of graph coloring and checks each solution for correctness and optimality. The algorithm introduces a special tree construction to avoid redundancy in enumerating solutions. The work by Brelaz (Brelaz, 1979) improves upon this algorithm by creating an initial coloring based on some clique in the graph and then considering assignments induced by this coloring. The work by Kubale and Kusz (Kubale \& Kusz, 1983) discusses the empirical performance of implicit enumeration algorithms, and later work by Kubale and Jackowski (Kubale \& Jackowski, 1985) augments traditional implicit enumeration techniques with more sophisticated backtracking methods.

Our work deals with solving graph coloring by reduction to another problem, in this case 0-1 ILP. This type of reduction has been discussed in the past, notably in the recent work by Mehrotra and Trick (Mehrotra \& Trick, 1996), which proposes an optimal coloring algorithm which expresses graph coloring using ILP-like constraints. It relies on an auxiliary independent set formulation, where each independent set in a graph is represented by a variable. There can be prohibitively many variables but in practical cases this number may be reduced by column generation, a method that first tries to solve a linear relaxation using a subset of variables and then adds more where needed. This approach inherently breaks problem symmetries, and thus rules out the use of SBPs as a way to speed up the search process. Our ILP construction differs considerably from the one described above, since it does not rely on an independent set formulation, but assigns colors to individual vertices by using indicator variables. The construction is described in more detail later in this section. Solving graph coloring by reduction allows exact solutions to be found by using SAT/0-1 ILP solvers as black boxes. Earlier work by Coudert (Coudert, 1997) demonstrated that finding exact solutions for application-derived graph coloring benchmarks often takes no longer than heuristic approaches, and that heuristic solutions may differ from the optimal value by as much as $100 \%$. Coudert (Coudert, 1997) proposes an algorithm that finds exact graph coloring solutions by solving the max-clique problem. The algorithm uses a technique called " $q$-color pruning", which assigns colors to vertices and systematically removes vertices that can be colored by $q$ colors, where $q$ is greater than a specified limit. 


\subsection{Breaking Symmetries in CSPs}

Several earlier works have addressed the importance of symmetry breaking in the search for solutions of CSPs. It has been shown (Krishnamurthy, 1985) that symmetry facilitates short proofs of propositions such as the pigeonhole principle, whereas pure-resolution proofs are necessarily exponential in size. Finding such proofs is, of course, a very difficult problem, but the performance of many CSP techniques can be lower-bounded by the best-case proof size. A typical approach to use symmetries is to prevent a CSP solver from considering redundant symmetric solutions. This is called symmetry-breaking and can be accomplished by adding constraints, often called symmetry-breaking predicates (SBPs). Static symmetrybreaking, such as the instance-independent constructions proposed in this work and the instance-dependent predicates from the literature (Aloul et al., 2003; Crawford et al., 1996), detects symmetries and adds SBPs during pre-processing and not when branching toward possible solutions. The Symmetry Breaking by Dominance Detection (SBDD) procedure described by Fahle in 2001 (Fahle, Schamberger, \& Sellmann, 2001) detects symmetric choice points during search. Each choice point generated by the search algorithm is checked against previously expanded search nodes. If the same or an equivalent choice point has been previously expanded, the choice point is not visited again. The global cut algorithm proposed by Focacci and Milano (Focacci \& Milano, 2001) records all nogoods found during search whose symmetric images should be pruned. This set of nogoods, called the "global cut seed" is used to generate global cut constraints that prune symmetric images for the entire search tree, while ensuring that correctness of the original constraints is not violated. Later work (Puget, 2002) has proposed improved methods for nogood recording. These works do not offer a systematic strategy for symmetry detection - they either require symmetries to be known or declared in advance, or record information during search that enables symmetry detection. Our work outlines and implements a complete strategy to detect and break symmetries automatically during pre-processing, so that a black-box solver can be used during search. This context is broader than those that justify the development of specialized solvers. On the other hand, our techniques do not conflict with dynamic symmetry-breaking and some of our results can potentially be reused in that context.

A promising new partially-dynamic approach to symmetry-breaking, called Group Equivalence (GE) trees is proposed by Roney et. al. (Roney-Dougal, Gent, Kelsey, \& Linton, 2004). This work aims to reduce the per-node overhead associated with dynamic approaches. A GE tree is constructed from a CSP with a symmetry group G such that the nodes of the tree represent equivalence classes of partial assignments under the group. This approach is illustrated by tracking value symmetries, i.e., simultaneous permutations of values in CSP variables. The work also shows that GE trees empirically outperform several well-known symmetry-breaking methodologies, such as SBDDs. In comparison, our work compares different ways to handle arbitrary compositions of variable and value symmetries (in graph coloring, value symmetries are seen at the specification level, whereas variable symmetries can only be seen in problem instances). To this end, our static techniques appear compatible rather than competing with the use of GE trees. There have also been many symmetry breaking approaches with particular relevance to graph coloring. Recent work by Gent (Gent, 2001) proposes constraints that break symmetry between "indistinguishable values", but does not evaluate them empirically. Like the lowest-index ordering (LI) constraints pro- 
posed by us in Section 3, these constraints also use the pre-existing sequential numbering of vertices in an instance of graph coloring to enforce distinctions between symmetric vertices. The construction appears complex compared to alternative SBPs and not as effective in our experiments as simpler constructions. Another related work is (Hentenryck, Agren, Flener, \& Pearson, 2003), which proposes a constant-time, constant-space algorithm for detecting and breaking value symmetries in a class of CSPs that includes graph coloring. More recently, Benhamou (Benhamou, 2004) discusses symmetry breaking for CSPs modeled using not-equals constraints (NECSP), and uses graph coloring as an illustrative example. The paper defines a sufficient condition for symmetry such that certain symmetries can be detected in linear time. The removal of these symmetries leads to considerable gains in backtracking search algorithms for NECSPs. In general, our empirical results, reported in Section 4, appear competitive with those for state-of-the-art dynamic approaches. However, designing the world's best graph-colorer is not the goal of our research. Instead, we focus on more efficient problem reductions to SAT and 0-1 ILP by improving symmetry-breaking. To ensure a broad applicability of our results, we treat SAT solvers as black boxes, and perform a comprehensive comparison of static SBPs and report empirical trends. While a more comprehensive comparison against existing graph coloring literature would be of great value, making it rigorous, conclusive and revealing requires that the best static and the best dynamic symmetry-breaking techniques are known. To this end, we speculate that a more likely winner would be a hybrid. Additional major issues to be resolved include the tuning of solvers to specific benchmarks (noted in the work by Kirovski and Potkonjak (Kirovski \& Potkonjak, 1998), differences in experimental setup, different software and hardware platforms, etc. Given that such a comparison is not completely in the scope of our work, it is better delegated to a dedicated publication. However, to demonstrate that our techniques are competitive with related work, we provide a comparison with the best results from recent literature (Benhamou, 2004; Coudert, 1997) in Section 4.3.

\subsection{SAT and 0-1 ILP}

One can solve the decision version of graph coloring by reducing it to Boolean satisfiability, and the optimization version by reduction to 0-1 ILP. The Boolean satisfiability (SAT) problem involves finding an assignment to a set of 0-1 variables that satisfies a set of constraints, called clauses, expressed in conjunctive normal form (CNF). A CNF formula on $n$ binary variables, $x_{1}, \ldots, x_{n}$ consists of a conjunction of clauses, $\omega_{1}, \ldots, \omega_{m}$. A clause consists of a disjunction of literals. A literal $l$ is an occurrence of a Boolean variable or its complement. The 0-1 ILP problem is closely related to SAT, and allows the use of pseudo-Boolean (PB) constraints, which are linear inequalities with integer coefficients that can be expressed in the normalized form (Aloul, Ramani, Markov, \& Sakallah, 2002) of: $a_{1} x_{1}+a_{2} x_{2}+\ldots a_{n} x_{n} \leq b$ where $a_{i}, b \in Z^{+}$and $x_{i}$ are literals of Boolean variables. ${ }^{1}$ In some cases a single $\mathrm{PB}$ constraint can replace an exponential number of CNF clauses (Aloul et al., 2002). In general, the efficiency of CNF reductions is encoding-dependent. Earlier work by Warners (Warners, 1998) shows that a linear-overhead conversion exists from linear inequalities with integer coefficients and 0-1 variables to CNF. However, CNF

1. Using the relations $(A x \geq b) \Leftrightarrow(-A x \leq-b)$ and $\overline{x_{i}}=\left(1-x_{i}\right)$, any arbitrary PB constraint can be expressed in normalized form with only positive coefficients. 
encodings which do not use this conversion may be less efficient. When converting CNF to PB, a single CNF constraint can always be expressed as a single 0-1 ILP constraint (by replacing disjunctions between literals in the constraint with ' + ' and setting the right-handside value as $\geq 1$ ). However, this may not always be suitable since certain operations, such as disjunction, implication and inequality are more intuitively expressed as CNF, and can be efficiently processed by SAT solvers such as Chaff (Moskewicz et al., 2001). A conversion to 0-1 ILP is more desirable for arithmetic operations, or "counting constraints", whose CNF equivalent requires polynomially many clauses (and exponentially many for some conversions). To maximize the advantages of both $\mathrm{CNF}$ and $\mathrm{PB}$ formats, most recent 0-1 ILP solvers such as PBS (Aloul et al., 2002) and Galena (Chai \& Kuehlmann, 2003) allow a formula to possess CNF and PB components. Additionally, 0-1 ILP solvers also provide for the solution of optimization problems. Subject to given constraints, one may request the minimization (or maximization) of an objective function which must be a linear combination of the problem variables.

Exact SAT solvers (Goldberg \& Novikov, 2002; Moskewicz et al., 2001; Silva \& Sakallah, 1999) are typically based on the original Davis-Logemann-Loveland (DLL) backtrack search algorithm (Davis, Logemann, \& Loveland, 1962). Recently, several powerful methods have been proposed to expedite the backtrack search algorithm, such as conflict diagnosis (Silva \& Sakallah, 1999) and watched literal Boolean constraint propagation (BCP) (Moskewicz et al., 2001). With these improvements, modern SAT solvers (Moskewicz et al., 2001; Goldberg \& Novikov, 2002) are capable of solving instances with several million variables and clauses in reasonable time. This increase in scalability and scope has enabled a number of SAT-based applications in various domains, including circuit layout (Aloul et al., 2003), microprocessor verification, symbolic model checking, and many others. More recent work has focused on extending advances in SAT to 0-1 ILP (Aloul et al., 2002; Chai \& Kuehlmann, 2003). In this work, we focus on solving instances of exact graph coloring by reduction to 0-1 ILP and the use of SBPs. Our choice of 0-1 ILP is motivated by the following reasons.

Firstly, 0-1 ILP permits the use of a more general input format than CNF, allowing greater efficiency in problem encoding, but at the same time is similar enough to SAT to allow improved methods for SAT-solving to be used without paying a penalty for generality. The specialized 0-1 ILP solvers PBS (Aloul et al., 2002) and Galena (Chai \& Kuehlmann, 2003) both propose sophisticated new techniques for 0-1 ILP that are based on recent decision heuristics (Moskewicz et al., 2001), conflict diagnosis and backtracking techniques (Silva \& Sakallah, 1999) for SAT solvers. As a result, they empirically perform better than both the generic ILP solver CPLEX (ILOG, 2000) and the leading-edge SAT solver zChaff on several DIMACS SAT benchmarks and application-derived instances such as FPGA routing instances from circuit layout. Also, since 0-1 ILP is an optimization problem, unlike SAT which is a decision problem, 0-1 ILP solvers possess the ability to maximize/minimize an objective function. They can, therefore, be directly applied to the optimization version of exact graph coloring, unlike pure CNF-SAT solvers that can only be used on the $k$-coloring decision variant. It is possible to solve the optimization version by repeatedly solving instances of the $k$-coloring using a SAT solver, with the value of $k$ being updated after each call. However, 0-1 ILP solvers do not require this extra step, and moreover tend to provide better performance than repeated calls to a SAT solver on many Boolean optimization problems (Aloul et al., 2002). 
It is possible to use a generic ILP solver, such as the commercial solver CPLEX (ILOG, 2000) instead of a specialized 0-1 ILP solver without any changes in problem formulation. However, Aloul et al. (Aloul et al., 2002) show that this generalization is not always desirable, particularly in the case of Boolean optimization problems such as Max-SAT. 0-1 ILP is also especially useful for evaluating the effectiveness of symmetry breaking for graph coloring, the primary purpose of this work. Detecting and breaking symmetries in SAT formulas has been shown to speed up the problem-solving process (Crawford et al., 1996; Aloul et al., 2003). Recently, symmetry breaking techniques for SAT have been extended to 0-1 ILP (Aloul et al., 2004), and have been shown to produce search speedups in this domain as well. However, a similar extension for non-binary variables for generic ILP does not presently exist. There is evidence (Aloul et al., 2002) that the advantages of symmetry breaking may depend on the actual algorithm used in the search. Specifically, results in the cited work suggest that the generic ILP solver CPLEX is actually slowed down by the addition of SBPs. Since CPLEX is a commercial tool and the algorithms used by it are not publicly known, it is difficult to pinpoint a reason for this disparity. However, our empirical results in Section 4 do bear out these observations. The remainder of this section discusses the reduction of graph coloring to 0-1 ILP and explains previous work in symmetry breaking in some detail.

\subsection{Detecting and Breaking Symmetries in 0-1 ILPs}

Previous work (Crawford et al., 1996; Aloul et al., 2003) has shown that breaking symmetries in CNF formulas effectively prunes the search space and can lead to significant runtime speedups. Breaking symmetries prevents symmetric images of search paths from being searched, thus pruning the search tree. The papers cited in this work all use variants of the approach first described by Crawford et al. (Crawford et al., 1996), which detects symmetries in a CNF formula using graph automorphism. The formula is expressed as an undirected graph such that the symmetry group of the graph is isomorphic to the symmetry group of the CNF formula. Symmetries induce equivalence relations on the set of truth assignments of the CNF formula. All assignments in an equivalence class result in the same truth value for the formula (satisfying or not). Therefore, it is only necessary to consider one assignment from each such class.

Techniques for symmetry breaking proposed in the literature follow the following steps: (i) construction of a colored graph from a CNF formula (ii) detection of symmetries in the graph using graph automorphism software (iii) use of the detected symmetries to construct symmetry breaking predicates (SBPs) that can be appended as additional clauses to the CNF formula (iv) solution of the new CNF formula thus created using a SAT solver. Crawford's construction (Crawford, 1992) uses 3 colors for vertices, one for positive literals, one for negative literals and a third for clauses. Edges are added between literals in a clause and the corresponding clause vertex, and between positive and negative literal vertices for Boolean consistency. As an optimization, binary clauses (with just two literals) are represented by adding an edge between the two involved literals, so an extra vertex is not needed. This is useful because the runtime of graph automorphism programs such as NAUTy (McKay, 1990) generally increases with the number of vertices in the graph. However, with this optimization Boolean consistency is not enforced, since binary clausal 
edges could be confused with Boolean consistency edges between positive and negative literals of the same variable. This may be improved by representing binary clausal edges as double edges (Crawford et al., 1996), thus distinguishing between the two edge types. However, NAUTY (and other graph automorphism programs) do not support the uses of double edges, so this construction is not very useful in practice. Furthermore, the cited constructions (Crawford, 1992; Crawford et al., 1996) do not allow detection of phase-shift symmetries, when a variable's positive literal is mapped to its negative literal and vice versa, since they color positive and negative literals differently. Our previous work (Aloul et al., 2003) improves upon these constructions by giving positive and negative literal vertices the same color, and allowing binary clauses and Boolean consistency edges to be represented the same way, i.e. a single edge between two literal vertices. Although this construction may allow spurious symmetries - when clause edges are mapped into consistency edges - this can occur only when a formula contains circular chains of implications over a subset of its variables. For example, given a subset of variables $x_{1} \ldots x_{n}$, such a chain is a collection of clauses $\left(y_{1} \Rightarrow y_{2}\right)\left(y_{2} \Rightarrow y_{3}\right) \ldots\left(y_{n-1} \Rightarrow y_{n}\right)$, where each $y_{i}$ is a positive or negative literal of $x_{i}$. These circular chains rarely occur in practice, and can be easily checked for. Therefore, the efficient graph construction described above can be used in most practical cases.

Graph automorphisms are detected in Crawford's work (Crawford et al., 1996) as well as our previous work (Aloul et al., 2003) using the program NAUTY (McKay, 1990), which is part of the GAP (Groups, Algebra and Programming) package. NAUTY accepts graphs in the GAP input format and returns a list of generators for the automorphism group (the term "generators" is used in a mathematical sense, the symmetry group partitions the set of vertex permutations for the graph into equivalence classes such that all permutations in the same class are equivalent. NAUTY returns the set of generators for this symmetry group). More recent work ((Aloul et al., 2003, 2004)) uses the automorphism program SAUCY (Darga et al., 2004), which is more efficient than NAUTY and can also process larger graphs with more vertices. After generators of the symmetry group are detected, symmetry breaking predicates are added to the instance in a pre-processing step. Crawford et al. (Crawford et al., 1996) propose the addition of SBPs that choose lexicographically smallest assignments (lex-leaders) from each equivalence class. We refer to such SBPs as instance-dependent SBPs, since the symmetries are first detected and then broken, and therefore the exact number and nature of SBPs added always depends on the connectivity of the graph itself. Although detecting symmetries is non-trivial, using modern software such as NAUTY and SAUCY the detection time is frequently insignificant when compared with SAT-solving time. Crawford et. al. (Crawford et al., 1996) construct lex-leader SBPs for the entire symmetry group, using the group generators returned by NAUTY. This type of symmetry breaking is complete. However, the approach used by Aloul et al. in TCAD 2003 (Aloul et al., 2003) shows that incomplete symmetry breaking, which breaks symmetries only between generators, is often effective in practice and much more efficient since it does not require the whole group to be reconstructed. The SBP construction proposed in the cited work (Aloul et al., 2003) is quadratic in the number of problem variables, compared with the earlier construction (Crawford et al., 1996), which could run to exponential size. This construction is further improved in the 2003 work by Aloul, Sakallah and Markov (Aloul et al., 2003), which describes efficient, tautology-free SBP construction, whose size is linear in the number of problem variables. Empirical results from both Crawford's work (Crawford et al., 1996) as 
well as the work in TCAD 2003 (Aloul et al., 2003) show that breaking symmetries produces large search speedups on a number of CNF benchmark families, including pigeonhole and Urquhart benchmarks, microprocessor verification, FPGA routing and ASIC global routing benchmarks from the VLSI domain.

Our work on symmetry breaking in SAT (Aloul et al., 2003) has also been extended to to optimization problems that include both CNF and PB constraints, and an objective function (Aloul et al., 2004). As before, symmetries are detected by reduction to graph automorphism. A PB formula for an optimization problem is represented by an undirected graph. Graph symmetries are detected using the graph automorphism tool SAUCY (Darga et al., 2004). Efficient symmetry breaking predicates (Aloul et al., 2003) are appended to the formula as CNF clauses. The empirical results for our work on symmetry breaking in 0-1 ILP (Aloul et al., 2004) show that the addition of symmetry breaking predicates to PB formulas results in considerable search speedups for the specialized 0-1 ILP solver PBS (Aloul et al., 2002). In this work, we use the above methodology (Aloul et al., 2004) for detecting and breaking instance-dependent symmetries in instances of graph coloring expressed as 0-1 ILP. These instance-dependent SBPs are compared with a number of instance-independent SBP constructions described in the next section.

Detecting and breaking symmetries in application-derived SAT instances amounts to a recovery of structure from the original application. The loss of structure during problem reductions is one reason why reduction-based techniques are often not competitive with domain-specific algorithms, and recent work on symmetry breaking is useful in this context. Other types of structure include clusters (Huang \& Darwiche, 2003; Aloul, Markov, \& Sakallah, 2004). Huang et al. (Huang \& Darwiche, 2003) propose an algorithm that detects clusters in SAT instances and uses them to produce variable orderings, and these structureaware orderings result in considerable empirical improvements with the SAT solver zChaff (Moskewicz et al., 2001).

\subsection{Reducing Graph Coloring to 0-1 ILP}

We express an instance of the minimal graph coloring problem as a 0-1 ILP optimization problem, consisting of (i) CNF and PB constraints that model the graph (ii) an objective function to minimize the number of colors used.

Consider a graph $G(V, E)$. Let $n=|V|$ be the number of vertices in $G$, and $m=|E|$ be the number of edges. An instance of the $K$-coloring problem for $G$ (i.e., can the vertices in $V$ be colored with $K$ colors) is formulated as follows.

- For each vertex $v_{i}, K$ indicator variables $x_{i, 1}, \ldots, x_{i, K}$, denote possible color assignments to $v_{i}$. Variable $x_{i, j}$ is set to 1 to indicate that vertex $v_{i}$ is colored with color $j$, and 0 otherwise

- For each vertex $v_{i}$, a PB constraint of the form $\sum_{j=1}^{K} x_{i, j}=1$ ensures that each vertex is colored with exactly one color.

- Each edge $e_{i}$ in $E$ connects two vertices $\left(v_{a}, v_{b}\right)$. For each edge $e_{i}$, we define CNF constraints of the form $\bigwedge_{j=1}^{K}\left(\overline{x_{a, j}} \vee \overline{x_{b, j}}\right)$ to specify that no two vertices connected by an edge can be given the same color. 
- To track used colors, we define $K$ new variables, $y_{1}, \ldots, y_{K}$. Variable $y_{i}$ is true if and only if at least one vertex uses color $i$. This is expressed using the following CNF constraints: $\bigwedge_{j=1}^{K}\left(y_{j} \Leftrightarrow\left(\bigvee_{i=1}^{n} x_{i, j}\right)\right)$.

- The optimization objective is to minimize the number of $y_{i}$ variables set to true, i.e. $\operatorname{MIN} \sum_{i=1}^{K} y_{i}$

The total number of variables in the formula is $n K+K$. The total number of constraints is computed as follows. There are totally $n$ 0-1 ILP constraints (one per vertex) to ensure that each vertex uses exactly one color. For each edge, there are $K$ CNF clauses specifying that the two vertices connected by that edge cannot have the same color, giving a total of $m K$ CNF clauses. There are an additional $n K$ CNF clauses ( $K$ per vertex) for setting indicator variables, and $K$ CNF clauses, one per color, to complete the iff condition for indicator variables. This gives a total of $K \cdot(m+n+1)$ CNF clauses and $n 0-1$ ILP constraints, plus one objective function, in the converted formula. For dense graphs, where $|E| \approx|V|^{2}$, the resulting formula size is quadratic in the number of vertices of the graph, but for sparser graphs it may be linear. A key observation is that instance-dependent symmetries in graph coloring survive the above reduction to 0-1 ILP. For instance-independent symmetries (i.e. permutations of colors) this is easy to see, since the ordering of colors can be changed without having any effect on the formula and producing the same set of constraints. For instance-dependent symmetries, consider two vertices $v_{a}$ and $v_{b}$ that are symmetric to each other and can be swapped in the original graph. Clearly, the constraints that specify that $v_{a}$ and $v_{b}$ must use exactly one color are interchangeable, as are the constraints that determine color usage based on the colors assigned to $v_{a}$ and $v_{b}$. It only remains to show that the connectivity constraints that control colors of vertices adjacent to $v_{a}$ and $v_{b}$ are also symmetric. This is clear from the fact that for every edge $E_{i}$ incident on $v_{a}$, there must be a corresponding edge $E_{j}$ incident on $v_{b}$ for the two vertices to be symmetric $\left(E_{i}\right.$ and $E_{j}$ can be the same edge). Therefore, for the set of $K \mathrm{CNF}$ clauses added to the formula to represent $E_{i}$, there must be a symmetric set of clauses added for $E_{j}$, and thus connectivity is preserved.

It is also clear that the 0-1 ILP formulation does not introduce spurious symmetries, i.e. any symmetry in the formula is a symmetry in the graph. A spurious symmetry arises when (i) variables of different types can be mapped into each other, e.g. vertex color variables are mapped to color usage indicator variables and (ii) variables of the same type are mapped into each other when the corresponding vertices are not actually symmetric. From the construction of the 0-1 ILP formula, it is clear that all $K$ variables per vertex that indicate a vertex's color can be permuted, as can the $K$ color usage variables, since these all appear in exactly the same constraints. This corresponds to the instance-independent symmetry colors in an instance of graph coloring can be arbitrarily permuted. However, vertex color variables appear in constraints restricting the number of colors a vertex can use and also in constraints that describe the connectivity of the graph, whereas color usage variables appear only in constraints that specify when they are set. Therefore, the two types of variables cannot map to one another. Since all constraints regarding color and connectivity of a vertex are written using all $K$ color variables for that vertex, these variables are symmetric to each other only in groups of $K$, i.e. if one such variable for a given vertex $v_{1}$ is symmetric to a variable for another vertex $v_{2}$, then all $K$ variables for $v_{1}$ and $v_{2}$ are correspondingly 
symmetric. Additionally, this symmetry between variables indicates a correspondence of clauses in which they occur. This is only possible if the vertices $v_{1}$ and $v_{2}$ are symmetric in terms of connectivity (instance-dependent symmetry). Thus, both types of symmetries are preserved during conversion to 0-1 ILP, and no false symmetries are added. Therefore, we can apply known techniques for symmetry detection in 0-1 ILP.

\section{Instance-Independent SBPs}

The question addressed in this work is whether instance-independent SBPs added during the reduction can provide even greater speedups, possibly by accelerating the detection of instance-dependent symmetries. To answer this question, we propose three provably correct SBP constructions of varying strength, and one heuristic that is intended to break a small number of symmetries with minimal overhead. Each construction is implemented and empirical results are reported in Section 4.

We use the following notation. Consider an instance of the $K$-coloring problem, which asks whether a graph $G(V, E)$ can be colored using $\leq K$ colors and minimizes the number of colors. Assume the colors are numbered $1 \ldots K$. We denote a valid color assignment by $\left(n_{1}, n_{2}, \ldots, n_{K}\right)$ where $n_{i}$ is the number of vertices colored with color $i$, and $|V|=\sum_{i=1}^{K} n_{i}$. Each $n_{i}$ in the color assignment denotes the cardinality of the independent set colored with color $i$. We are not concerned with the actual composition of the independent sets here, since that is an instance-dependent issue. Instance-independent symmetries are only the arbitrary permutations of colors between different independent sets.

The effects of each proposed construction are illustrated using the example in Figure 1. The figure is an example of the 4-coloring problem on a graph with four vertices. Part (a) of the figure shows the graph to be colored. For visual clarity, part (b) shows color patterns corresponding to the different color numbers. It is clear from the figure that the vertices $V_{1}, V_{2}$ and $V_{3}$ form a clique, and must use different colors. However, $V_{4}$ can be given the same color as either $V_{1}$ or $V_{2}$, and therefore only 3 colors are needed for this instance. The instance can be partitioned into independent sets in two ways: $\left\{\left\{V_{1}, V_{4}\right\},\left\{V_{2}\right\},\left\{V_{3}\right\}\right\}$ and $\left\{\left\{V_{1}\right\},\left\{V_{2}, V_{4}\right\},\left\{V_{3}\right\}\right\}$. Our SBPs do not actually address how the independent sets are composed, because this is an instance-dependent issue. However, given any partition of independent sets, colors can be arbitrarily permuted between sets in the partition. The instance-independent SBPs proposed here restrict this permutation. In the examples below, we assume the first partition of independent sets i.e. $\left\{\left\{V_{1}, V_{4}\right\},\left\{V_{2}\right\},\left\{V_{3}\right\}\right\}$. Results are proved with respect to the permutation of colors for this partition.

\subsection{Null-Color Elimination (NU)}

Consider a $K$-coloring problem with colors $1 \ldots K$ for a graph $G(V, E)$. Assume that $G$ can be minimally colored with $K-1$ colors. Consider an optimal solution where color $i$ is not used: $\left(n_{1}, n_{2}, . . n_{i-1}, 0, n_{i+1}, \ldots, n_{K}\right)$. This assignment is equivalent to another assignment,

$$
\left(n_{1}^{\prime}, n_{2}^{\prime}, . . n_{j-1}^{\prime}, 0, n^{\prime}{ }_{j+1} \ldots n_{K}^{\prime}\right)
$$

where $i \neq j$ and $n_{i}^{\prime}=n_{j}$. For example, the assignment $(1,0,2,3)$ is equivalent to $(1,3,2,0)$, $(0,1,2,3),(1,2,0,3)$. This is due to the existence of null colors, which create symmetries in 


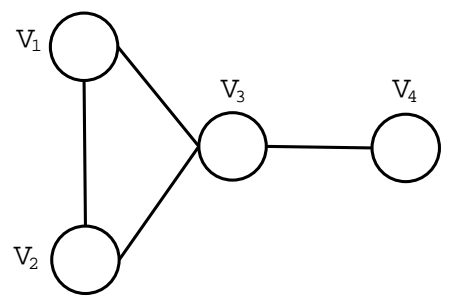

(a)

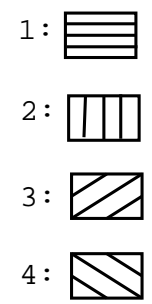

(b)
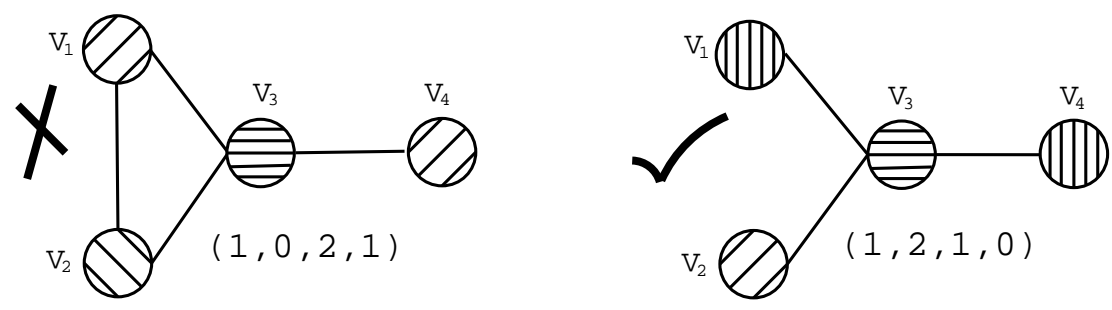

(c)
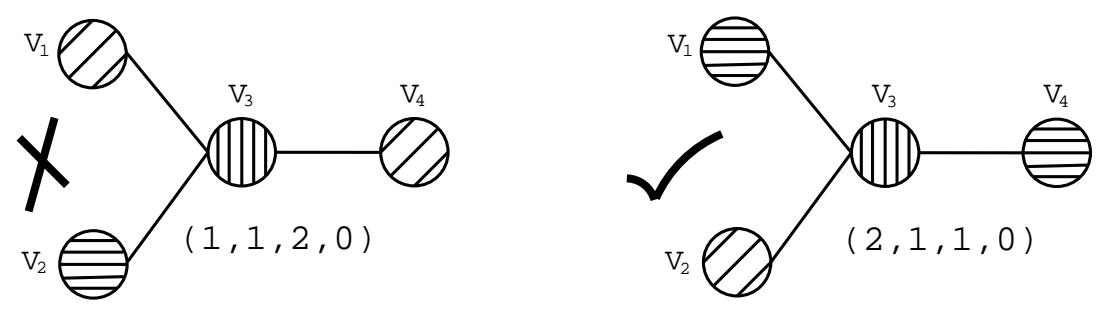

(d)
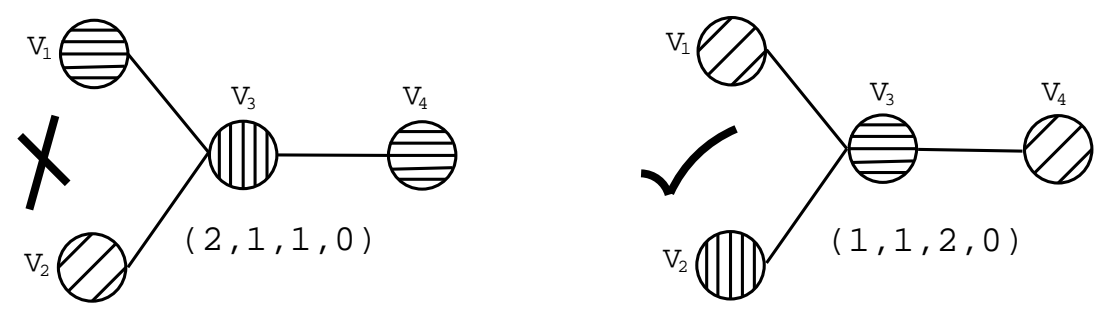

(e)

Figure 1: Instance-independent symmetry breaking predicates (SBPs). Part (a) shows the original graph with no vertices colored. Part (b) shows the color key. Part (c) shows how nullcolor SBPs prevent color 4 from being used. Part (d) shows how cardinality based SBPs assign colors in the order of independent set sizes, allowing fewer assignments than nullcolor SBPs. Part (e) demonstrates how lowest-index ordered SBPs break symmetries that are undetected by other types of SBPs. 
an instance of $K$-coloring because any color can be swapped with a null color. Null colors are extraneous because they are not actually required to color any vertices, and so can be inserted anywhere in a solution, as seen above. We propose a construction that enforces an ordering on null colors: null colors may appear only at the end of a color assignment, after all non-null colors. This is implemented by adding $K-1 \mathrm{CNF}$ constraints of the form: $y_{k+1} \Rightarrow y_{k}$ for $1 \leq k \leq K-1$, to the original formulation. In the example above, only one of the four symmetric assignments $(1,3,2,0)$ would be allowed under this construction. Since our ILP formulation defines and sets the $K$ indicator variables that track color usage, it is extremely easy to enforce null color elimination as described above. The SBPs require the addition of no extra variables and only $K-1$ new CNF clauses.

We prove that the proposed construction is correct. Assume that under the original formulation, an optimal solution for graph $G(V, E)$ uses $m$ colors. Assume that this solution contains null colors and non-null colors, and with null-color elimination, there is a different optimal solution that uses $m^{\prime}$ colors, where $m \neq m^{\prime}$. The only colors used in this solution are $1 \ldots m^{\prime}$, since null colors cannot occur before non-null colors. Since our construction adds SBPs without changing the original constraints, any legal solution that satisfies the SBPs will satisfy all constraints in the original formulation. The solution to the original satisfies all constraints in the new formulation except the SBPs. If $m<m^{\prime}$, we can re-order the solution so that all null colors are placed last. This will satisfy all SBPs and use $m$ colors, where $m<m^{\prime}$, violating the assumption that the $m^{\prime}$-color solution was optimal. If $m^{\prime}<m$, we already have a solution that satisfies all the original constraints and uses fewer colors, which again violates assumptions of optimality.

An illustration of the use of NU predicates for the example in Figure 1 (a) is shown in Figure 1 (c). The figure shows two valid minimal-color assignments to the graph vertices in the example. The assignment on the left uses colors 1, 3 and 4, while the one on the right uses colors 1, 2 and 3. The assignments are symmetric but under NU predicates only the right-hand side assignment is permissible.

\subsection{Cardinality-Based Color Ordering (CA)}

Null-color elimination is useful only in cases where null colors exist. For a $K$-coloring problem where all colors are needed, the construction breaks no symmetries. Even when null colors exist, several symmetries go undetected. In the first example from above, nullcolor elimination permits six symmetric color assignments $(1,2,3,0),(1,3,2,0),(2,1,3,0)$, $(2,3,1,0)(3,2,1,0)$ and $(3,1,2,0)$. This is because restrictions are placed on null colors, but the ordering of non-null colors is unrestricted. A stronger construction would distinguish between the independent sets themselves. We propose an alternative construction, which assigns colors based on the cardinality of independent sets. This subsumes null-color elimination, since null colors can be viewed as coloring sets of cardinality 0 . The cardinality rule is implemented as follows: the largest independent set is assigned the color 1 , the second-largest the color 2 , etc. In the example above, only the assignment $(3,2,1,0)$ is valid. This is enforced by adding $K-1 \mathrm{~PB}$ constraints of the form: $\sum_{i=1}^{n} x_{i, k} \geq \sum_{i=1}^{n} x_{i, k+1}$, where $1 \leq k \leq K-1$. Again, this construction is fairly simple to implement, requiring only $K-1$ additional constraints. However, these are 0-1 ILP constraints with multiple 
variables, unlike the simple CNF implication clauses between two variables used for the NU predicates. Thus, there is some overhead for greater completeness.

We prove the CA construction correct as follows. Assume an optimal solution under this construction uses $m<K$ colors: $\left(n_{1}, n_{2}, \ldots, n_{m}\right)$, where $\left(n_{1} \geq n_{2} \ldots \geq n_{m}\right)$. Colors $>m$ are not used on any vertex, Assume there exists an optimal solution to the original formulation that uses $m^{\prime}$ colors: $\left(n_{1}^{\prime}{ }_{1}, n_{2}^{\prime}, \ldots, n_{m^{\prime}}^{\prime}\right.$ ), (where $n_{1}^{\prime}$, etc. are not arranged in descending order). Without loss of generality, assume that $m^{\prime}<m$. We can sort the numbers $n_{1}^{\prime}, \ldots, n_{m^{\prime}}^{\prime}$ and reassign colors in descending order. We would have a solution with $m^{\prime}$ colors satisfying cardinality constraints. However, $m^{\prime}<m$, which is not possible if the $m$-color solution was optimal. A similar argument applies when $m<m^{\prime}$.

For the example from Figure 1 (a), only the largest independent set under the partition we are considering, i.e. $\left\{V_{1}, V_{4}\right\}$ can be given color 1 . Therefore, the assignment on the right of Figure 1 (c), which assigns the largest set color 2 and is correct under NU predicates, is incorrect under the CA construction. The left-hand side of Figure 1 (d) shows another assignment that is correct under NU predicates but incorrect under CA predicates, since it assigns the set $\left\{V_{1}, V_{4}\right\}$ color 3 . A correct assignment, shown on the right-hand side of Figure 1 (d), gives the largest set color 1 and since both the other sets have one element each, they can each be assigned either color 2 or color 3 . Thus, several symmetric assignments which survive NU predicates are prohibited under this construction.

\subsection{Lowest Index Color Ordering (LI)}

While more complete than NU predicates, CA predicates do not break symmetries when different independent sets have the same cardinality. Consider a graph $G$ where $V=$ $\left\{v_{1}, \ldots, v_{8}\right\}$, and an optimal solution, satisfying cardinality-based ordering, that partitions $V$ into 4 independent sets: $S_{1}=\left\{v_{4}, v_{6}, v_{7}\right\}, S_{2}=\left\{v_{1}, v_{5}\right\}, S_{3}=\left\{v_{3}, v_{8}\right\}, S_{4}=\left\{v_{2}\right\}$. A solution that assigns colors 2 and 3 to $S_{2}$ and $S_{3}$ is symmetric to one that assigns colors 2 and 3 to $S_{3}$ and $S_{2}$. Both are legal under cardinality-based ordering. In order to completely break symmetries, it is not adequate to distinguish between sets solely on the basis of cardinality (unless no two sets have the same cardinality). It is necessary to construct SBPs based on the actual composition of sets in a partition, which is unique. However, the distinctions that we make on the basis of composition are not to be confused with instance-dependent SBPs, since our construction is implemented before the symmetries in an instance are known, and regardless of its actual composition. The SBPs here specify broad guidelines for the coloring of independent sets that are applicable to all graphs. To improve upon cardinality-based ordering, we propose a set of predicates to enforce the lowest-index ordering (LI). Consider all vertices with color $i$, and find the lowest index $j_{i}$ among those. We require that the lowest indices for each color be ordered. This constraint can be enforced by adding inequalities for colors with adjacent numbers.

Note that each color has a unique lowest-index vertex — otherwise some vertex would have to be colored with two colors. In the above example, the only color assignment compatible with the partitioning of vertices into independent sets is: color 1 to $S_{1}, 2$ to $S_{3}$, 3 to $S_{4}$, and 4 to $S_{2}$.

To evaluate the strength of this symmetry-breaking technique, consider an arbitrary coloring and a color permutation that remains a symmetry after the LI constraints are 
imposed. If any colors are permuted simultaneously on all vertices, this will permute the lowest indices for those colors. Since all lowest indices are different, their ordering is completely determined by the ordering of colors, and thus the color permutation we chose must be the identity permutation. In other words, no instance-independent symmetries remain after symmetry-breaking with LI.

We implement lowest-index color ordering as follows. For each vertex $v_{i}$, we declare a new set of $K$ variables, $V_{i, 1}, \ldots V_{i, K}$. Variable $V_{i, k}$ being set implies that vertex $v_{i}$ is the lowest-index vertex colored with color $k$. This is enforced by the following CNF constraints: $V_{i, k} \Rightarrow\left(\bigwedge_{j=1}^{i-1} \overline{V_{j, k}}\right)$. Also, exactly one $V_{i, j}$ variable must be true for every color used. Therefore, we add the constraints: $y_{k} \Rightarrow \bigvee_{i=1}^{n} V_{i, k}$, where $1 \leq k \leq K, y_{k}$ are the variables that indicate color $k$ is used, and $n=|V|$ from Section 2. Finally, the following CNF clause is added for each $V_{i, k}$ to ensure lowest-index ordering: $V_{i, k} \Rightarrow\left(\bigvee_{j=i+1}^{n} V_{j, k-1}\right)$, Since the LI ordering completely breaks symmetries between independent sets, it subsumes earlier constructions. However, it does come at an added cost. While the NU and CA constructions required no new variables and only $K-1$ constraints, the LI construction requires $n K$ new variables and an additional $2 n K$ CNF clauses, which is almost double the size of the original formula.

The LI construction can be proved correct by the same means as the CA construction. Given an optimal assignment of colors to independent sets, we can sort the independent sets in order of lowest-index vertex and assign colors from 1 to $K$ accordingly, without affecting correctness.

Figure 1 (e) illustrates the effect of LI SBPs on the example in Figure 1 (a). The graph on the left, which is shown as being correct for CA predicates in Figure 1 (d) is incorrect under the LI construction, because the lowest-index vertex with color $2\left(V_{3}\right)$ does not have a higher index than the lowest-index vertex with color 3, which is $V_{2}$. The graph on the right shows the correct assignment, which under LI predicates is the only permissible assignment for the partition $\left\{\left\{V_{3}\right\},\left\{V_{2}\right\},\left\{V_{1}, V_{4}\right\}\right\}$.

In addition to being very complex, LI predicates are so rigid that they obscure symmetries of the original instance. For example, in Figure 1 (a), it is easily seen that the vertices $V_{1}$ and $V_{2}$ are symmetric and can be permuted with no effect on the resulting graph. This symmetry is instance-dependent - it is decided by the way $V_{1}$ and $V_{2}$ are connected. Without the addition of any SBPs, it is apparent that under any legal coloring of the graph, the colors given to $V_{1}$ and $V_{2}$ can be swapped regardless of how $V_{3}$ and $V_{4}$ are colored. The NU predicates preserve this symmetry, since they are only concerned with null colors which by definition could not be used on $V_{1}$ and $V_{2}$. The CA predicates also preserve the symmetry since $V_{1}$ and $V_{2}$ can be interchangeably used in any independent set, and swapping them between sets would not have any effect on the cardinality of the sets. However, under the LI predicates, an independent set containing $V_{1}$ must always be given a higher-numbered color than a set containing $V_{2}$, and the two cannot be interchanged. If $V_{1}$ was given any color other than the highest color in use, there would exist some independent set whose color index was 1 greater than the color assigned to $V_{1}$, and for this set, the lowest-index predicate would not be satisfied. Thus, LI predicates actually destroy any vertex permutations in the graph. This is seen in our empirical results in Section 4, where the addition of 
LI SBPs leaves no symmetries in any of the benchmarks. This is unusual because ordinarily benchmarks of reasonable size would contain at least some vertex permutations.

\subsection{Selective Coloring (SC)}

It is noticeable that the ILP formulation and constraints can be very complex for more complete SBPs, such as the LI predicates above, which introduce several additional variables and clauses. This raises the question of whether such a complex construction is actually counterproductive - it may break symmetries, but require so much effort during search that the benefit of complete symmetry breaking is lost. To investigate this, we also propose a simple "heuristic" construction to break some symmetries between vertices while adding almost no additional constraints. To impact as many vertices as possible, we find the vertex $v_{l}$ with the largest degree of all vertices in the graph. We then color $v_{l}$ with color 1 . This is achieved by simply adding the unary clause $x_{l, 1}$. We search $v_{l}$ 's neighbors to find the vertex $v_{l^{\prime}}$ with the highest degree out of all vertices adjacent to $v_{l}$. We color $v_{l^{\prime}}$ with color 2 , by adding the unary clause $x_{l^{\prime}, 2}$. This construction has the effect of simplifying color assignment for all vertices adjacent to $v_{l}$ and $v_{l^{\prime}}$. No vertex adjacent to $v_{l}$ can be colored color 1 , and no vertex adjacent to $v_{l^{\prime}}$ can be colored color 2. Moreover, all vertices in an independent set with $v_{l}\left(v_{l^{\prime}}\right)$ must be colored color 1 (color 2). If $v_{l}$ and $v_{l^{\prime}}$ have sufficiently large degree, this construction can restrict many vertex assignments. An even stronger construction would be to find a triangular clique and fix colors for all three vertices in it; however, clique finding is complicated and some graphs may not possess any such cliques. We refer to this construction as selective coloring.

The extent to which selective coloring breaks symmetries is instance-dependent. It fails to completely break symmetries for almost all graphs. However, it is a simple construction, adding just two constraints as unary clauses. These are easily resolved in pre-processing by most SAT solvers, so any symmetry breaking achieved by this construction has virtually no overhead.

We note that all instance-independent predicates defined here are only concerned with symmetries between colors, which exist in any instance of graph coloring. However, additional instance-independent symmetries may be introduced during the reduction to graph coloring for certain applications. For example, in the radio frequency assignment applica-

tion from Section 2, adding all possible bipartite edges between cliques for adjacent regions will result in symmetries between vertices in these cliques. Additional predicates can be added to instances from this application to break these symmetries.

\section{Empirical Results}

This section describes our experimental setup, empirical results, and performance compared with related work.

\subsection{Experimental Setup}

We used 20 medium-sized instances from the DIMACS graph coloring benchmark suite. We briefly describe each family of benchmarks used below. 
Breaking Instance-Independent Symmetries in Exact Graph Coloring

- Random graphs. Benchmarks with randomly created connections between vertices, named DSJ

- Book graphs. Edges represent interaction between characters in a book. There are four such benchmarks: anna, david, huck, jean

- Mileage graphs. These represent distances between cities on a map, and are named miles

- Football game graphs. Indicate relationships between teams that must play each other in college football games. In the tables these are referred to as games

- $n$-queens graphs. Instances of the $n$-queens problem, named queen

- Register allocation graphs. Represent the register allocation problem for different systems. We use two families in this work, named mulsol, zeroin

- Mycielski graphs. Instances of triangle-free graphs based on the Mycielski (Mycielski, 1955) transformation, called myciel

Table 1 gives the name, size (number of vertices and edges) and the chromatic number for each benchmark. We use a maximum value of $K=20$ for $K$-coloring. For benchmarks with chromatic number $>20$, we do not report the chromatic number.

Our problem formulation with a fixed $K$ is application-driven. Indeed, in many domains it is only useful to find the exact chromatic number when it is below a well-known threshold. For example, in graph coloring instances from register allocation, there cannot be more colors than processor registers. PC processors often have 32 registers, and high-end CPUs may have more. However, realistic graphs are relatively sparse and have low chromatic numbers. On the other hand, processors embedded in cellular phones, automobiles and point-of-sale terminals may have very few registers, leading to tighter constraints on acceptable chromatic numbers. The value $K=20$ used in our experiments is in no way special, but the results achieved with it are representative of other results. Also, while we apply the $K=20$ bound to all instances here to study trends, more reasonable bounds can be determined on a per-instance basis using the following simple procedure.

1. Apply any heuristic for min-coloring to determine a feasible upper bound

2. If the value is relatively small, perform linear search by incrementally tightening the color constraint, otherwise perform binary search

Benchmark graphs are transformed into instances of 0-1 ILP using the conversion described in Section 2. To solve instances of 0-1 ILP, we used the academic 0-1 ILP solvers PBS (Aloul et al., 2002), Galena (Chai \& Kuehlmann, 2003), and Pueblo (Sheini, 2004), and also the commercial ILP solver CPLEX version 7.0. Pueblo is more recent than PBS and Galena, and incorporates Pseudo-Boolean (PB) learning based on ILP cutting-plane techniques. We use a later version of PBS, PBS II, that enhances the original PBS algorithms (Aloul et al., 2002) with learning techniques from the Pueblo solver (Sheini, 2004). We do not include the results with the original version of PBS that are reported in (Ramani, 


\begin{tabular}{|l||c|c|c|}
\hline Instance & $\# \mathrm{~V}$ & $\# \mathrm{E}$ & $K$ \\
\hline anna & 138 & 986 & 11 \\
david & 87 & 812 & 11 \\
DSJC125.1 & 125 & 1472 & 5 \\
DSJC125.9 & 125 & 13922 & $>20$ \\
games120 & 120 & 1276 & 9 \\
huck & 74 & 602 & 11 \\
jean & 80 & 508 & 10 \\
miles250 & 128 & 774 & 8 \\
mulsol.i.2 & 188 & 3885 & $>20$ \\
mulsol.i.4 & 185 & 3946 & $>20$ \\
myciel3 & 11 & 20 & 4 \\
myciel4 & 23 & 71 & 5 \\
myciel5 & 47 & 236 & 6 \\
queen5_5 & 25 & 320 & 5 \\
queen6_6 & 36 & 580 & 7 \\
queen7_7 & 49 & 952 & 7 \\
queen8_12 & 96 & 2736 & 12 \\
zeroin.i.1 & 211 & 4100 & $>20$ \\
zeroin.i.2 & 211 & 3541 & $>20$ \\
zeroin.i.3 & 206 & 3540 & $>20$ \\
\hline
\end{tabular}

Table 1: DIMACS graph coloring benchmarks

Aloul, Markov, \& Sakallah, 2004), since it has been retired by the newer version. However, in the Appendix we report detailed results for $n$ - queens instances using the older version of PBS along with results for the other solvers, for the sake of a more detailed study. PBS II is implemented in $\mathrm{C}++$ and compiled using $\mathrm{g}++$. Galena and Pueblo binaries were provided by the authors. PBS was run using the variable state independent decaying sum (VSIDS) decision heuristic option (Moskewicz et al., 2001). Galena was run using its default options of linear search with cardinality reduction (CARD) learning. All experiments are run on Sun-Blade-1000 workstations with 2GB RAM, CPUs clocked at $750 \mathrm{MHz}$ and the Solaris operating system. Time-out limits for all solvers are set at 1000 seconds.

We use the symmetry breaking flow first proposed in our earlier work (Aloul et al., 2004 ) to detect and break symmetries in our original ILP formulation from Section 2. This flow uses the tool ShatTer (Aloul et al., 2003), which uses the SAUCY (Darga et al., 2004) graph automorphism program and the efficient SBP construction from (Aloul et al., 2003). We also check for unbroken symmetries in formulations produced by each of the instanceindependent constructions described in Section 3. Our runtimes for symmetry detection and for solving the reduced 0-1 ILP problems are reported in the next section.

\subsection{Runtimes for Symmetry Detection and 0-1 ILP Solving}

Table 2 shows symmetry detection results and runtimes. The numbers reported in the table are sums of individual results for all 20 benchmarks used. We report statistics as sums because reporting results for all of SBPs on all benchmarks would be space-consuming, and 


\begin{tabular}{|c|c|c|c|c|c|c|}
\hline \multirow{2}{*}{$\begin{array}{l}\text { SBP } \\
\text { Type }\end{array}$} & \multicolumn{3}{|c|}{ CNF Stats } & \multicolumn{3}{|c|}{ Sym. Stats (SAUCY) } \\
\hline & $\# \mathrm{~V}$ & \#CL & $\# \mathrm{~PB}$ & $\# \mathrm{~S}$ & $\# \mathrm{G}$ & Time \\
\hline no SBPs & $437 \mathrm{~K}$ & 777505 & 3193 & $1.1 \mathrm{e}+168$ & 994 & 185 \\
\hline $\mathrm{NU}$ & $437 \mathrm{~K}$ & 777885 & 3193 & $5.0 \mathrm{e}+149$ & 614 & 49 \\
\hline $\mathrm{CA}$ & $437 \mathrm{~K}$ & 777505 & 3630 & $5.0 \mathrm{e}+149$ & 614 & 49 \\
\hline LI & $870 \mathrm{~K}$ & 4019980 & 3193 & $2.0 \mathrm{e}+01$ & 0 & 84 \\
\hline $\mathrm{SC}$ & $437 \mathrm{~K}$ & 777545 & 3193 & $3.0 \mathrm{e}+164$ & 941 & 167 \\
\hline $\mathrm{NU}+\mathrm{SC}$ & $437 \mathrm{~K}$ & 777925 & 3193 & $5.0 \mathrm{e}+148$ & 597 & 47 \\
\hline
\end{tabular}

Table 2: CNF formula sizes, symmetry detection results and runtimes, totaled for 20 benchmarks from Table 1, with $K=20$. NU = null-color elimination; $\mathbf{C A}=$ cardinality-based; $\mathbf{L I}=$ lowest-index; $\mathrm{SC}=$ selective coloring. For the LI SBPs, one instance of the "do-nothing" symmetry is counted in each case, giving a total of 20 symmetries and 0 generators. SAUCY is run on an Intel Xeon dual processor at $2 \mathrm{GHz}$ running RedHat Linux 9.0.

would also not illustrate trends as clearly. This work is concerned with characterizing the broad impact of symmetry breaking. However, we show detailed results for the queens instances in the Appendix.

The first column in the table indicates the type of construction: we use no SBPs for the basic formulation, NU for null-color elimination, CA for cardinality-based ordering, LI for lowest-index ordering, and SC for selective coloring (the last row shows NU and SC in combination). The next three columns show the number of variables, CNF clauses, and $\mathrm{PB}$ constraints in the problems. The last three columns show the number of symmetries, number of symmetry generators, and symmetry detection runtimes for SAUCY. Henceforth, we will refer to instance-dependent SBPs as external, because they are added to an instance after symmetries are detected and are not part of the problem formulation. The top row is separated from the bottom 5 rows because it represents statistics without instanceindependent SBPs. We observe that adding instance-independent SBPs during problem formulation does cut down the symmetry detection runtime considerably. SAUCY has a total runtime of 185 seconds when no instance-independent SBPs are added, but its runtimes with NU, CA, LI and NU + SC constructions are much smaller. Only the SC construction has a comparable runtime because it is a heuristic and breaks very few symmetries. The columns showing numbers of symmetries and generators support this observation: the NU, $\mathrm{CA}, \mathrm{LI}$ and $\mathrm{NU}+\mathrm{SC}$ constructions all have far fewer symmetries than the top row, but the SC construction has almost the same number. For these benchmarks, the LI construction, breaks all symmetries, even instance-dependent vertex permutations that may exist in a graph. SAUCY reports finding no symmetries for this construction (except one instance of the do-nothing symmetry for each graph, which is trivial). However, SAUCY runtimes for this construction are larger than for the NU, CA and NU + SC constructions (85 seconds to approximately 49 seconds) even though there are no symmetries in the instances after LI 


\begin{tabular}{|c|c|c|c|c|c|c|c|c|c|c|c|c|c|c|c|c|}
\hline \multirow{3}{*}{$\begin{array}{l}\text { SBP } \\
\text { Type }\end{array}$} & \multicolumn{4}{|c|}{ PBS II, PB Learning } & \multicolumn{4}{|c|}{ CPLEX } & \multicolumn{4}{|c|}{ Galena } & \multicolumn{4}{|c|}{ Pueblo } \\
\hline & \multicolumn{2}{|c|}{ Orig. } & \multicolumn{2}{|c|}{ w/i.-d. SBPs } & \multicolumn{2}{|c|}{ Orig. } & \multicolumn{2}{|c|}{ w/i.-d. SBPs } & \multicolumn{2}{|c|}{ Orig. } & \multicolumn{2}{|c|}{ w/i.-d. SBPs } & \multicolumn{2}{|c|}{ Orig. } & \multicolumn{2}{|c|}{ w/i.-d. SBPs } \\
\hline & Tm. & $\# \mathrm{~S}$ & Tm. & $\# \mathrm{~S}$ & Tm. & $\# \mathrm{~S}$ & Tm. & \#S & Tm. & \#S & Tm. & $\# \mathrm{~S}$ & Tm. & \#S & Tm. & $\# \mathrm{~S}$ \\
\hline no SBPs & $17 \mathrm{~K}$ & 3 & $4.2 \mathrm{~K}$ & 16 & $6.3 \mathrm{~K}$ & 14 & $13 \mathrm{~K}$ & 7 & $1.7 \mathrm{~K}$ & 2 & $3 \mathrm{~K}$ & 17 & $18 \mathrm{~K}$ & 3 & $1.6 \mathrm{~K}$ & 19 \\
\hline $\mathrm{NU}$ & $8.2 \mathrm{~K}$ & 13 & $7.5 \mathrm{~K}$ & 13 & $5.9 \mathrm{~K}$ & 15 & $6.5 \mathrm{~K}$ & 15 & $8.3 \mathrm{~K}$ & 11 & $6.7 \mathrm{~K}$ & 11 & $9.1 \mathrm{~K}$ & 12 & $8.3 \mathrm{~K}$ & 13 \\
\hline $\mathrm{CA}$ & $13 \mathrm{~K}$ & 6 & $12 \mathrm{~K}$ & 8 & $11 \mathrm{~K}$ & 11 & $11 \mathrm{~K}$ & 10 & $19 \mathrm{~K}$ & 1 & $17 \mathrm{~K}$ & 3 & $9 \mathrm{~K}$ & 12 & $10 \mathrm{~K}$ & 12 \\
\hline LI & $15 \mathrm{~K}$ & 6 & $15 \mathrm{~K}$ & 6 & $16 \mathrm{~K}$ & 4 & $16 \mathrm{~K}$ & 4 & $15 \mathrm{~K}$ & 5 & $15 \mathrm{~K}$ & 5 & $16 \mathrm{~K}$ & 5 & $16 \mathrm{~K}$ & 5 \\
\hline $\mathrm{SC}$ & $14 \mathrm{~K}$ & 6 & 65 & 20 & $5.3 \mathrm{~K}$ & 15 & $12 \mathrm{~K}$ & 8 & $16 \mathrm{~K}$ & 4 & 94.4 & 20 & $15 \mathrm{k}$ & 5 & $2.1 \mathrm{~K}$ & 18 \\
\hline $\mathrm{NU}+\mathrm{SC}$ & $6.9 \mathrm{~K}$ & 14 & $6.8 \mathrm{~K}$ & 14 & $4.5 \mathrm{~K}$ & 16 & $6.4 \mathrm{~K}$ & 14 & $6.1 \mathrm{~K}$ & 14 & $6.1 \mathrm{~K}$ & 14 & $7.3 \mathrm{~K}$ & 13 & $7.1 \mathrm{~K}$ & 13 \\
\hline
\end{tabular}

Table 3: Runtimes and number of solutions found before and after SBPs are added for all constructions using PBS II (with PB learning), CPLEX, Galena and Pueblo; all experiments are run on SunBlade 1000 workstations. Timeouts for all solvers were set at $1000 \mathrm{~s}$. The maximum color limit is set at 20 , instances with $k>20$ are unsatisfiable under these formulations. This is not a comparison of solvers. We solve ILP formulations with equal optimal values using different solvers to weed out solver-specific issues. Best results for a given solver are shown in boldface. In the entries, $\mathrm{K}$ denotes multiples of 1000 s seconds rounded to the nearest integer.

predicates are added. A likely reason for this is the sharp increase in instance size caused by the LI construction. In general, the SC construction has very little effect on the number of symmetries - when used by itself, it leaves most symmetries intact, and when used with the NU construction, the improvement over the NU construction alone is very small.

Table 3 shows the effect of symmetry breaking on runtimes of PBS II (Aloul et al., 2002), CPLEX (ILOG, 2000), Galena (Chai \& Kuehlmann, 2003) and Pueblo (Sheini, 2004). The first column in the table specifies the construction type, followed by the total runtime for each solver (with and without the addition of instance-independent SBPs) and the number of instances solved for the construction. For each solver, the best performance among all configurations (largest number of instances solved and corresponding runtime) is boldfaced. Results are given first for the new version of PBS, PBS II based on (Sheini, 2004), followed by CPLEX, Galena and Pueblo. Runtimes for the older version of PBS can be obtained from our earlier work (Ramani et al., 2004). To compare performance of an individual solver for different constructions, observe the runtime and solution entries for different rows in the same column, and to compare performance for different solvers on the same constructions, observe numbers for the same row across all columns. We observe the following trends.

1. All benchmarks possess a large number of symmetries. Different instance-independent SBPs achieve varying degrees of completeness: the lowest-index ordering (LI) breaks all symmetries in the benchmarks used, while the selective coloring (SC) SBP breaks the fewest symmetries. SAUCY runtimes for residual symmetry detection after the addition of instance-independent SBPs are highest for the no SBPs construction and the SC construction, since they possess the largest numbers of symmetries

2. For the case where no SBPs of any kind are added, CPLEX performs well, solving 14 out of 20 instances within the time limit. However, PBS II, Galena and Pueblo perform poorly - Galena solves only 2 instances and PBS II and Pueblo each solve 3 
3. PBS II, Galena and Pueblo benefit considerably from instance-dependent symmetry breaking. When instance-dependent SBPs are used without any of the instanceindependent constructions we propose, PBS II solves 16 instances within the time limit, while Galena and Pueblo solve 17 and 19 instances respectively. However, CPLEX is hampered by the addition of instance-dependent SBPs, and solves only 7 instances in this case

4. Adding only instance-independent SBPs improves performance for all specialized 01 ILP solvers over the no-SBP version. The best performance for PBS II, Galena and Pueblo is seen for the NU + SC construction - PBS II and Galena solve 14 instances, and Pueblo solves 13. For CPLEX, the NU + SC construction shows marginal improvement over the no-SBPs case (16 instances are solved), but the more complex constructions, CA and LI, actually undermine performance - CPLEX solves only 4 instances with the LI construction. In general, complex SBP constructions perform much worse than simple ones. PBS II, Pueblo and Galena also perform poorly with the CA and LI constructions - Galena solves only 1 instance with the CA construction with no help from instance-dependent SBPs, and very few instances are solved with the LI construction for any solver

5. Adding instance-independent SBPs alone does not solve as many instances as adding instance-dependent SBPs to the SBP-free formulation. The best performance seen with instance-independent SBPs is 14 instances solved, by Galena and PBS II, and 16 instances solved by CPLEX, with the NU + SC construction. When instancedependent SBPs are added PBS II and Galena solve all 20 instances with the SC construction. The CA and LI constructions leave very few (or none at all) symmetries to be broken by instance-dependent SBPs. Consequently, there is almost no difference in results with and without instance-dependent SBPs for these constructions. However, they do not achieve the same performance improvements as instance-dependent SBPs, due to their size and complexity

6. Using instance-dependent SBPs in conjunction with the SC construction is useful. With this combination, PBS II and Galena solve all 20 instances within the time limit, and Pueblo solves 18. Runtime is also considerably improved for PBS II and Galena - PBS II solves all 20 instances in a total of 65 seconds, and Galena in 94.4 seconds. The best overall performance, in terms of number of solutions and runtime, is seen with this combination. In general, however, the $\mathrm{SC}$ construction is not dominant on its own. Results for the SC construction alone are very similar to results with no SBPs, and results for the NU + SC combination are very similar to those achieved by using only NU SBPs. The SC construction is effective at "boosting" the performance of other constructions

7. The three specialized 0-1 ILP solvers - PBS II, Galena and Pueblo, exhibit the same performance trends with respect to the constructions used, and their performances are all comparable, in terms of both the number of solutions found and runtime This indicates that the variations in performance are due to the different SBPs, not due to differing solver implementations. All solvers are independent implementations based 


\begin{tabular}{|c|c|c|c|c|c|c|c|c|c|c|c|c|c|c|c|c|}
\hline \multirow{3}{*}{$\begin{array}{l}\text { SBP } \\
\text { Type }\end{array}$} & \multicolumn{4}{|c|}{ PBS II, PB Learning } & \multicolumn{4}{|c|}{ CPLEX } & \multicolumn{4}{|c|}{ Galena } & \multicolumn{4}{|c|}{ Pueblo } \\
\hline & \multicolumn{2}{|c|}{ Orig. } & \multicolumn{2}{|c|}{ w/i.-d. SBPs } & \multicolumn{2}{|c|}{ Orig. } & \multicolumn{2}{|c|}{ w/i.-d. SBPs } & \multicolumn{2}{|c|}{ Orig. } & \multicolumn{2}{|c|}{ w/i.-d. SBPs } & \multicolumn{2}{|c|}{ Orig. } & \multicolumn{2}{|c|}{ w/i.-d. SBPs } \\
\hline & Tm. & $\# \mathrm{~S}$ & Tm. & $\# \mathrm{~S}$ & Tm. & $\# \mathrm{~S}$ & Tm. & $\# \mathrm{~S}$ & Tm. & \#S & Tm. & $\# \mathrm{~S}$ & Tm. & \#S & Tm. & \#S \\
\hline No SBPs & $18 \mathrm{~K}$ & 2 & $6.2 \mathrm{~K}$ & 14 & $11 \mathrm{~K}$ & 9 & $8.2 \mathrm{~K}$ & 12 & $19 \mathrm{~K}$ & 1 & $9.1 \mathrm{~K}$ & 11 & $19 \mathrm{~K}$ & 1 & $7.5 \mathrm{~K}$ & 13 \\
\hline NU & $9.2 \mathrm{~K}$ & 12 & $7.9 \mathrm{~K}$ & 13 & $11 \mathrm{~K}$ & 9 & $12 \mathrm{~K}$ & 8 & $10 \mathrm{~K}$ & 10 & $7.6 \mathrm{~K}$ & 13 & $11 \mathrm{~K}$ & 11 & $9.5 \mathrm{~K}$ & 11 \\
\hline $\mathrm{CA}$ & $13 \mathrm{~K}$ & 7 & $13 \mathrm{~K}$ & 9 & $13 \mathrm{~K}$ & 9 & $14 \mathrm{~K}$ & 8 & $19 \mathrm{~K}$ & 1 & $17 \mathrm{~K}$ & 4 & $11 \mathrm{~K}$ & 11 & $13 \mathrm{~K}$ & 8 \\
\hline LI & $15 \mathrm{~K}$ & 5 & $15 \mathrm{~K}$ & 5 & $19 \mathrm{~K}$ & 2 & $19 \mathrm{~K}$ & 2 & $16 \mathrm{~K}$ & 5 & $16 \mathrm{~K}$ & 5 & $17 \mathrm{~K}$ & 3 & $17 \mathrm{~K}$ & 3 \\
\hline $\mathrm{SC}$ & $15 \mathrm{~K}$ & 5 & $5.3 \mathrm{~K}$ & 15 & $10 \mathrm{~K}$ & 10 & $12 \mathrm{~K}$ & 9 & $16 \mathrm{~K}$ & 4 & $5.3 \mathrm{~K}$ & 15 & $16 \mathrm{~K}$ & 4 & $6.0 \mathrm{~K}$ & 15 \\
\hline $\mathrm{NU}+\mathrm{SC}$ & $7.1 \mathrm{~K}$ & 13 & $7.0 \mathrm{~K}$ & 13 & $9.7 \mathrm{~K}$ & 11 & $9.9 \mathrm{~K}$ & 11 & $9.2 \mathrm{~K}$ & 12 & $6.9 \mathrm{~K}$ & 14 & $8.0 \mathrm{~K}$ & 13 & $7.4 \mathrm{~K}$ & 13 \\
\hline
\end{tabular}

Table 4: Total runtimes and number of solutions found before and after SBPs are added for all constructions using PBS II (with PB learning), CPLEX, Galena and Pueblo. The experimental setup is the same as that used in Table 3 but with a color limit of $K=30$. Best results for a solver are boldfaced. Fewer instances are solved than in Table 3 because the higher color limit results in larger and potentially more difficult instances.

on the same algorithmic framework (the Davis-Logemann-Loveland backtrack search procedure), but PBS II and Galena also have learning capabilities

8. Adding instance-dependent SBPs to any construction usually adversely affects the performance of CPLEX. This has been previously noted in other work (Aloul et al., 2004). Since the CPLEX algorithms and implementation are not available in the public domain, it is difficult to account for this effect. However, PBS and Galena with symmetry breaking significantly outperform CPLEX without symmetry breaking

9. We report results as the sum of runtimes for all instances to illustrate trends. On a per-instance basis, the same trends are displayed. For example, for the no-SBPs case in the top row, PBS II solves 3 instances and Galena solves 2, but the two instances solved by Galena are among those solved by PBS II. In general, the same instances tend to be "easy" or "difficult" for the 0-1 ILP solvers, although CPLEX behaves differently. An example of this behavior for the queens family of instances is illustrated in the Appendix

Overall, the results suggest that for graph coloring, adding instance-independent SBPs alone is not competitive with the use of instance-dependent SBPs alone. The best results are achieved using a combination of both types, and even here, the instance-independent SBPs used are the most simple variety. This is true even when symmetry detection runtimes are taken into consideration. We attribute this result to the complexity of instance-independent SBPs we use, and also to the fact that improvements in graph automorphism software (Darga et al., 2004) have greatly reduced the overhead of detecting symmetries by reduction to graph automorphism. Previously, for static approaches that require symmetries to be detected and broken in advance, the task of symmetry detection was often a bottleneck that could actually take longer than the search itself. With this bottleneck removed, the advantages of static symmetry breaking - simple predicates that address specific symmetries rather than complex constructions that alter the problem specification considerably - are more clearly illustrated. Even among instance-independent predicates, simple constructions are more effective than complex ones. As we have noted in Section 3, simple constructions 
like NU and SC add very few additional constraints and do not alter the original problem greatly. However, the CA and LI constructions add many more constraints, which may confuse the specialized 0-1 ILP solvers.

It is important to note that color permutations, while instance-independent, do appear at the instance-specific level. Thus, the symmetries targeted by instance-independent predicates are a subset of those targeted by instance-dependent predicates. Our instanceindependent constructions are not intended to cover a different set of symmetries, but rather to break some of the same symmetries during problem formulation, thus reducing or eliminating the overhead of any instance-dependent methods that may follow. The fact that this strategy is not successful suggests that, for the same set of symmetries, the instancedependent predicates we use are more efficient and easier for solvers to tackle.

To verify our claims about performance trends, we show results for an additional set of experiments with increased color limit $K=30$ in Table 4 . The instances are re-formulated with $K=30$ and with different SBP constructions. This experiment is intended to verify trends from the $K=20$ case, and to investigate whether instances with chromatic number $>20$, that are unsatisfiable in the first case, can be colored with $\leq 30$ colors. Results from Table 4 validate our observations from Table 3 - the best results for PBS II, Galena and Pueblo are again achieved with the NU + SC (with no instance-dependent SBPs) and SC (with instance-dependent SBPs) constructions. However, with this formulation fewer instances are solved than for the $K=20$ case, possibly because the $K=30$ limit results in larger instances. Also, for instances whose chromatic number is much closer to 30 than 20 , it may be harder to prove optimality, whereas proving unsatisfiability for the $K=20$ experiments may be simpler.

\subsection{Comparison with Related Work}

Here, we discuss the empirical performance of our approach when compared with related work (Coudert, 1997; Benhamou, 2004). We note that both cited works describe algorithms specifically developed for graph coloring, and the search procedures cannot be used to solve other problems. Our approach, on the other hand, solves hard problems by reduction to generic problems such as SAT or 0-1 ILP, and this work on graph coloring can be viewed as a case study. Consequently, we use problem-specific knowledge only during the actual problem formulation (instance-independent SBPs are also added during reduction), but not during search itself. This may be useful for applications where problem-specific solvers cannot be developed or acquired due to limited resources. Our goal is to determine whether symmetry breaking can improve the performance of reduction-based methods, which are traditionally not competitive with problem-specific methods. Thus, while our techniques may not be superior to all problem-specific solvers on all instances, we hope to show reasonably strong performance over a broad spectrum of instances.

Common data points between our work and Coudert's (Coudert, 1997) include instances of queens, myciel and DSCJ125.1. Referring to our detailed results for queens instances in the Appendix, we note that our runtimes are competitive with those of Coudert's algorithm - for example, on queen5_5, both algorithms have a runtime of 0.01s. On larger instances, however, our runtimes are somewhat slower. On the myciel instances, we obtain the best results with the Pueblo solver and the SC predicates, with runtimes of 0.01, 0.06, and 1.80s 
on mycie13, 4, and 5, compared with $0.01,0.02$ and 4.17 for Coudert's algorithm. Therefore, it appears that our approach is competitive on these common data points. Moreover, other studies (Kirovski \& Potkonjak, 1998) have observed that Coudert's work does not provide results for several hard real-world problem classes, particularly those where modeling results in dense graphs. Our work is more general, and cannot be biased to favor certain types of graphs.

The algorithm described by Benhamou (Benhamou, 2004) shows very competitive runtimes on a number of DIMACS benchmarks, particularly instances of register allocation. For example, the DSJC125.1 instance is solved by Benhamou's algorithm in 0.01 seconds, while the best time achieved by us is 1.12 seconds, using the Pueblo solver with only instance-dependent SBPs. However, we note that Benhamou's algorithm determines the upper limit for the chromatic number $K$ using more instance-specific knowledge, for example, for DSCJ125.1, it is set at $K=5$. We solve all instances with $K=20$, which may be too large a limit in some cases. The value of $K$ affects the size of the resulting 0-1 ILP reductions and SBPs, which is likely to affect runtime. We also note that the DIMACS benchmarks used in the cited work (Benhamou, 2004) are primarily register allocation and randomly generated instances, whereas we achieve reasonably good performance on a wide variety of benchmark applications. Moreover, Benhamou's approach relies on modeling graph coloring as a not-equals CSP, which does not bode well for generality. Many CSPs cannot be modeled using only not-equals constraints. Additionally, the symmetry detection, breaking and search procedures described in that work are specific to graph coloring, whereas our work can be extended to several other problems, only requiring a reduction to SAT/0-1 ILP.

\section{Conclusions}

Our work shows that problem reduction to 0-1 ILP is a viable method for optimally solving combinatorial problems without investing in specialized solvers. This approach is likely to be even more successful as the efficiency of 0-1 ILP solvers improves in the future, and as they are able to better handle problem structure. In particular, problem reductions may produce highly-structured instances making the ability to automatically detect and exploit structure very important. In the case of graph coloring we demonstrate that a generic, publicly-available symmetry breaking flow from our earlier work (Aloul et al., 2004) significantly improves empirical results in conjunction with the academic 0-1 ILP solvers PBS II, a new version of the solver PBS (Aloul et al., 2002), Galena (Chai \& Kuehlmann, 2003) and Pueblo (Sheini, 2004). All specialized 0-1 ILP solvers significantly outperform the commercial generic ILP solver CPLEX 7.0 when symmetry-breaking is used. The performance of CPLEX actually deteriorates when SBPs are added, and on the original instances with no SBPs, CPLEX is able to solve more instances than the 0-1 ILP solvers. However, the best performance overall is obtained with the 0-1 ILP solvers on instances with SBPs added. Although our techniques are tested on standard DIMACS benchmarks instances, we note that the symmetry-breaking flow described here can be applied to graph coloring instances from any application.

We are particularly interested in comparing strategies for breaking symmetries that are present in every ILP instance produced by problem reduction (instance-independent sym- 
metries). Such symmetries may be known even before the first instances of the original problem are delivered (i.e., symmetries may be detected at the specification level), and one has the option to use them during problem reduction. Intuitively, this may prevent discovering these symmetries in every instance and thus improve the overall CPU time. To this end, we propose four constructions for instance-independent symmetry breaking predicates (SBPs). These constructions vary in terms of strength and completeness. Our goal in experiments was to compare the performance of the four instance-independent SBP constructions relative to each other, as well as to assess their performance when compared with instance-dependent SBPs. Instance-independent SBPs have the advantage of not requiring the additional step of symmetry detection, since they are part of the problem specification. Additionally, they are designed with more information about the problem itself, and their effect on solutions is clear - for example, we know that null-color elimination will force all the lower-numbered colors to be used in a solution. Instance-dependent SBPs are detected and added automatically on the 0-1 ILP reduction of an instance without any understanding of their significance. On the other hand, instance-dependent constructions are less complex and result in more compact predicates. Our empirical data indicate that simplicity of construction is a more powerful factor in determining performance - instance-dependent SBPs consistently outperform instance-independent SBPs, and the most complete and complex instance-independent constructions (LI) are actually the weakest in performance. It is clear from our results that symmetry breaking itself is useful in graph coloring: adding instance-dependent SBPs always speeds up search over the no-SBPs case. It is likely that instance-independent SBPs are less successful due to their complex construction. Simpler instance-independent constructions (NU, SC) outperform the more complex ones (CA, LI). It is well known that the syntactic structure of CNF and PB constraints may dramatically affect the efficiency of SAT and ILP solvers. Shorter clauses and PB constraints are much preferable as they are easier to resolve against other constraints, and are more useful to the learning strategies employed by exact SAT solvers. Another factor that gives instancedependent SBPs the advantage is the ease of symmetry detection, which was previously a bottleneck. Due to improved software (Darga et al., 2004), the overhead of symmetry detection via reduction to graph automorphism in SAT/0-1 ILP instances is almost negligible.

We also show that the three specialized 0-1 ILP solvers, PBS II, Galena and Pueblo, all exhibit similar performance trends for different constructions. This indicates that performance is not decided by solver-specific issues, but by the difficulty of the instances and the SBPs added to them. CPLEX does not display the same behavior as the other solvers, and is in fact slowed down by the addition of instance-dependent SBPs and by several instance-independent constructions. CPLEX is a commercial solver for generic ILP problems, and its algorithms and decision heuristics are likely to be very different than those used by academic solvers. However, since details about CPLEX are not publicly available, it is not possible to accurately explain its behavior. We do note that while CPLEX does not appear to benefit from symmetry breaking, its performance on the reduced instances with no SBPs of any kind is superior to the 0-1 ILP solvers. However, once SBPs are added the specialized solvers solve more instances than CPLEX in less time.

In the context of generic search and combinatorial optimization problems defined in the NP-spec language (Cadoli et al., 1999), our empirical data suggest that new theoretical breakthroughs are required to make use of instance-independent symmetries during problem 
reductions to SAT or 0-1 ILP. At our current level of understanding, the simple strategy of processing instance-independent and instance-dependent symmetries together produces smallest runtimes for graph coloring benchmarks. Our current and future work is focused on developing more effective SBPs for this problem, and also investigating the utility of symmetry breaking for other hard search problems. Moreover, while our work uses instanceindependent predicates only for color symmetries, our results and analysis may have broader scope, for example, in applications such as radio frequency assignment (Section 2) where symmetries are introduced during the reduction to graph coloring and are likely to be preserved during future reductions. The issues involved in using instance-dependent vs. instance-independent SBPs are very relevant to such applications.

\section{Acknowledgments}

This work was funded in part by NSF ITR Grant \#0205288. Also, we thank Donald Chai and Andreas Kuehlmann from UC Berkeley for providing us with binaries of the Galena solver, and Hossein Sheini for providing us with the binaries for Pueblo.

\section{Appendix A: Performance Analysis on Queens Instances}

This section provides a more detailed discussion of our results on individual benchmarks in the queens family of instances. The problem posed by queens instances is whether queens can be placed on an $n \times m$ chessboard without conflicts. The instances we use in our experiments are queens $5 \times 5,6 \times 6,7 \times 7$ and $8 \times 12$. Table 5 shows results for the queens family. Results are shown for every instance with no SBPs, with each of the four constructions NU, CA, LI and SC, and with the NU + SC combination. All constructions are tested with and without instance-dependent SBPs as before. We report results for the original version of PBS, from (Aloul et al., 2002), and for PBS II, CPLEX, Galena and Pueblo as in Section 4. Experiments are run on Sun Blade 1000 workstations as before. In the table, we report solver runtime if an instance is solved, and T/O for a timeout at 1000 seconds. The best results for a solver on a particular instance are boldfaced.

While there is greater variation when considering performance on a per-instance basis, the table largely reflects the same trends reported in Section 4. For example, when no instance-dependent SBPs are used, PBS, PBS II, Galena and Pueblo all largely perform best with the NU + SC construction. When instance-dependent SBPs are added, the best performance is seen with the $\mathrm{SC}$ construction in most cases. CPLEX does not display the same behavior as the other solvers, and its performance clearly deteriorates when instancedependent SBPs are added to any construction. A similar effect has been observed in related work (Aloul et al., 2004). Results for the original version of PBS (Aloul et al., 2002), which could not be included in Section 4, have been added in this section. It can be seen that PBS follows the same trends as PBS II, Galena and Pueblo, reinforcing our claim that this behavior is not solver-dependent. 


\begin{tabular}{|c|c|c|c|c|c|c|c|c|c|c|c|}
\hline \multirow{3}{*}{$\begin{array}{l}\text { Inst. } \\
\text { Name }\end{array}$} & \multirow{3}{*}{$\begin{array}{l}\text { SBP } \\
\text { Type }\end{array}$} & \multicolumn{2}{|c|}{ PBS } & \multicolumn{2}{|c|}{ PBS II } & \multicolumn{2}{|c|}{ CPLEX } & \multicolumn{2}{|c|}{ Galena } & \multicolumn{2}{|c|}{ Pueblo } \\
\hline & & \multicolumn{2}{|c|}{$\begin{array}{l}\text { Inst.-dep. } \\
\text { SBPs used? }\end{array}$} & \multicolumn{2}{|c|}{$\begin{array}{c}\text { Inst.-dep. } \\
\text { SBPs used? }\end{array}$} & \multicolumn{2}{|c|}{$\begin{array}{l}\text { Inst.-dep. } \\
\text { SBPs used? }\end{array}$} & \multicolumn{2}{|c|}{$\begin{array}{c}\text { Inst.-dep. } \\
\text { SBPs used? }\end{array}$} & \multicolumn{2}{|c|}{$\begin{array}{c}\text { Inst.-dep. } \\
\text { SBPs used? }\end{array}$} \\
\hline & & No & Yes & No & Yes & $\mathrm{No}$ & Yes & No & Yes & No & Yes \\
\hline \multirow{6}{*}{ queen5_5 } & no SBPs & $\overline{\mathrm{T} / \mathrm{O}}$ & 0.19 & 34.52 & 0.04 & 1.11 & 643.93 & 83.06 & 0.35 & 203.09 & 0.01 \\
\hline & $\mathrm{NU}$ & 1.84 & $\mathrm{~T} / \mathrm{O}$ & 0.01 & 0.02 & 1.38 & 23.67 & 0.21 & 0.27 & 0.08 & 0.1 \\
\hline & $\mathrm{CA}$ & $\mathrm{T} / \mathrm{O}$ & $\mathrm{T} / \mathrm{O}$ & 0.31 & 0.24 & 39.2 & 2.76 & $\mathrm{~T} / \mathrm{O}$ & $\mathrm{T} / \mathrm{O}$ & 0.14 & 0.52 \\
\hline & LI & 135 & 134.71 & 1.48 & 1.48 & 262.96 & 217.21 & 5.4 & 5.4 & 8.48 & 8.48 \\
\hline & $\mathrm{SC}$ & 15.99 & 0.19 & 0.15 & 0.07 & 0.45 & 229.79 & 0.29 & 0.29 & 0.25 & 0.19 \\
\hline & $\mathrm{NU}+\mathrm{SC}$ & 8.63 & 12.34 & 0 & 0.01 & 0.83 & 0.88 & 0.3 & 1 & 0.06 & 0.07 \\
\hline \multirow{6}{*}{ queen6_6 } & no SBPs & $\mathrm{T} / \mathrm{O}$ & 3.61 & $\mathrm{~T} / \mathrm{O}$ & 0.21 & $\mathrm{~T} / \mathrm{O}$ & $\mathrm{T} / \mathrm{O}$ & $\mathrm{T} / \mathrm{O}$ & 0.87 & $\mathrm{~T} / \mathrm{O}$ & 0.49 \\
\hline & $\mathrm{NU}$ & 331.63 & 521.12 & 56.63 & 13.59 & $\mathrm{~T} / \mathrm{O}$ & $\mathrm{T} / \mathrm{O}$ & 192.17 & 19.11 & 123.99 & 18.88 \\
\hline & $\mathrm{CA}$ & $\mathrm{T} / \mathrm{O}$ & $\mathrm{T} / \mathrm{O}$ & 50.6 & 780.57 & $\mathrm{~T} / \mathrm{O}$ & $\mathrm{T} / \mathrm{O}$ & $\mathrm{T} / \mathrm{O}$ & $\mathrm{T} / \mathrm{O}$ & 196.94 & 80.53 \\
\hline & LI & $\mathrm{T} / \mathrm{O}$ & $\mathrm{T} / \mathrm{O}$ & $\mathrm{T} / \mathrm{O}$ & $\mathrm{T} / \mathrm{O}$ & $\mathrm{T} / \mathrm{O}$ & $\mathrm{T} / \mathrm{O}$ & $\mathrm{T} / \mathrm{O}$ & $\mathrm{T} / \mathrm{O}$ & $\mathrm{T} / \mathrm{O}$ & $\mathrm{T} / \mathrm{O}$ \\
\hline & $\mathrm{SC}$ & $\mathrm{T} / \mathrm{O}$ & 0.58 & $\mathrm{~T} / \mathrm{O}$ & 0.1 & 242.79 & $\mathrm{~T} / \mathrm{O}$ & $\mathrm{T} / \mathrm{O}$ & 1.0 & $\mathrm{~T} / \mathrm{O}$ & 0.32 \\
\hline & $\mathrm{NU}+\mathrm{SC}$ & 2.89 & 1.72 & 1.4 & 0.63 & 95.91 & $\mathrm{~T} / \mathrm{O}$ & 11.19 & 1.05 & 4.85 & 2.64 \\
\hline \multirow{6}{*}{ queen7_7 } & no SBPs & $\mathrm{T} / \mathrm{O}$ & 36.56 & $\mathrm{~T} / \mathrm{O}$ & 1.79 & 243.3 & $\mathrm{~T} / \mathrm{O}$ & $\mathrm{T} / \mathrm{O}$ & $\mathrm{T} / \mathrm{O}$ & $\mathrm{T} / \mathrm{O}$ & 1.13 \\
\hline & $\mathrm{NU}$ & 0.45 & 3.29 & 36.31 & 24.74 & 119.16 & 459.44 & 56.6 & 147.52 & 9.59 & 15.49 \\
\hline & $\mathrm{CA}$ & $\mathrm{T} / \mathrm{O}$ & $\mathrm{T} / \mathrm{O}$ & $\mathrm{T} / \mathrm{O}$ & $\mathrm{T} / \mathrm{O}$ & 271.2 & $\mathrm{~T} / \mathrm{O}$ & $\mathrm{T} / \mathrm{O}$ & $\mathrm{T} / \mathrm{O}$ & 692.67 & 150.86 \\
\hline & LI & $\mathrm{T} / \mathrm{O}$ & $\mathrm{T} / \mathrm{O}$ & 53.3 & 53.4 & $\mathrm{~T} / \mathrm{O}$ & $\mathrm{T} / \mathrm{O}$ & 78.85 & 78.8 & 212.18 & 213.8 \\
\hline & $\mathrm{SC}$ & $\mathrm{T} / \mathrm{O}$ & 8.42 & 38.57 & 0.85 & 38.04 & $\mathrm{~T} / \mathrm{O}$ & $\mathrm{T} / \mathrm{O}$ & 1.33 & 217.82 & 1.23 \\
\hline & $\mathrm{NU}+\mathrm{SC}$ & 5.65 & 38.07 & 4.37 & 5.73 & 119.7 & $\mathrm{~T} / \mathrm{O}$ & 17.46 & 5.16 & 25.73 & 14.04 \\
\hline \multirow{6}{*}{ queen8_12 } & no SBPs & $\mathrm{T} / \mathrm{O}$ & 1.31 & $\mathrm{~T} / \mathrm{O}$ & 0.52 & $\mathrm{~T} / \mathrm{O}$ & $\mathrm{T} / \mathrm{O}$ & $\mathrm{T} / \mathrm{O}$ & $\mathrm{T} / \mathrm{O}$ & $\mathrm{T} / \mathrm{O}$ & $\mathrm{T} / \mathrm{O}$ \\
\hline & $\mathrm{NU}$ & $\mathrm{T} / \mathrm{O}$ & $\mathrm{T} / \mathrm{O}$ & $\mathrm{T} / \mathrm{O}$ & $\mathrm{T} / \mathrm{O}$ & $\mathrm{T} / \mathrm{O}$ & $\mathrm{T} / \mathrm{O}$ & $\mathrm{T} / \mathrm{O}$ & 138.61 & $\mathrm{~T} / \mathrm{O}$ & $\mathrm{T} / \mathrm{O}$ \\
\hline & $\mathrm{CA}$ & $\mathrm{T} / \mathrm{O}$ & $\mathrm{T} / \mathrm{O}$ & $\mathrm{T} / \mathrm{O}$ & $\mathrm{T} / \mathrm{O}$ & $\mathrm{T} / \mathrm{O}$ & $\mathrm{T} / \mathrm{O}$ & $\mathrm{T} / \mathrm{O}$ & $\mathrm{T} / \mathrm{O}$ & $\mathrm{T} / \mathrm{O}$ & $\mathrm{T} / \mathrm{O}$ \\
\hline & LI & $\mathrm{T} / \mathrm{O}$ & $\mathrm{T} / \mathrm{O}$ & $\mathrm{T} / \mathrm{O}$ & $\mathrm{T} / \mathrm{O}$ & $\mathrm{T} / \mathrm{O}$ & $\mathrm{T} / \mathrm{O}$ & $\mathrm{T} / \mathrm{O}$ & $\mathrm{T} / \mathrm{O}$ & $\mathrm{T} / \mathrm{O}$ & $\mathrm{T} / \mathrm{O}$ \\
\hline & $\mathrm{SC}$ & $\mathrm{T} / \mathrm{O}$ & 1.05 & $\mathrm{~T} / \mathrm{O}$ & 0.47 & $\mathrm{~T} / \mathrm{O}$ & $\mathrm{T} / \mathrm{O}$ & $\mathrm{T} / \mathrm{O}$ & 1.9 & $\mathrm{~T} / \mathrm{O}$ & 0.98 \\
\hline & $\mathrm{NU}+\mathrm{SC}$ & $\mathrm{T} / \mathrm{O}$ & $\mathrm{T} / \mathrm{O}$ & 787.26 & 780.14 & $\mathrm{~T} / \mathrm{O}$ & $\mathrm{T} / \mathrm{O}$ & 52.1 & 53.63 & $\mathrm{~T} / \mathrm{O}$ & $\mathrm{T} / \mathrm{O}$ \\
\hline
\end{tabular}

Table 5: Detailed results for queens instances. For each instance, we show results for the solvers PBS, PBS II, CPLEX, Galena and Pueblo. All solvers are run on SunBlade 1000 workstations. Instances are tested with no instance-independent SBPs, with each of the four proposed constructions in Section 3 and with a combination of the NU and SC constructions. All instance-independent SBPs are tested alone and with instance-dependent SBPs added. The table shows the runtime for a given instance under different construction. $\mathrm{T} / \mathrm{O}$ indicates a timeout at 1000 seconds. Best results for a given solver on each instance are shown in boldface.

\section{References}

Aloul, F. A., Markov, I. L., \& Sakallah, K. A. (2003). Shatter: Efficient symmetry-breaking for boolean satisfiability. In International Joint Conference on Artificial Intelligence, pp. 271-282.

Aloul, F. A., Markov, I. L., \& Sakallah, K. A. (2004). MINCE: A static global variableordering heuristic for sat search and bdd manipulation. Journal of Universal Computer Science (JUCS), 10, 1562-1596.

Aloul, F. A., Ramani, A., Markov, I. L., \& Sakallah, K. A. (2002). Generic ILP versus specialized 0-1 ILP: An update. In International Conference on Computer-Aided Design, pp. 450-457.

Aloul, F. A., Ramani, A., Markov, I. L., \& Sakallah, K. A. (2003). Solving difficult instances 
of boolean satisfiability in the presence of symmetry. IEEE Transactions on CAD, 22, 1117-1137.

Aloul, F. A., Ramani, A., Markov, I. L., \& Sakallah, K. A. (2004). Symmetry-breaking for pseudo-boolean formulas. In Asia-Pacific Design Automation Conference, pp. 884887.

Aragon, C. R., Johnson, D. S., McGeoch, L. A., \& Schevon, C. (1991). Optimization by simulated annealing: An experimental evaluation; part ii, graph coloring and number partitioning. Operations Research, 39, 378-406.

Benhamou, B. (2004). Symmetry in not-equals binary constraint networks. In Workshop on Symmetry in CSPs, pp. 2-8.

Brelaz, D. (1979). New methods to color vertices of a graph. Communications of the ACM, 22, 251-256.

Brown, R. J. (1972). Chromatic scheduling and the chromatic number problem. Management Science, 19, 451-463.

Cadoli, M., \& Mancini, T. (2003). Detecting and breaking symmetries on specifications. In The Third Annual Workshop on Symmetry in Constraint Satisfaction Problems (SymCon), pp. 13-26.

Cadoli, M., Palopoli, L., Schaerf, A., \& Vasileet, D. (1999). NP-SPEC: An executable specification language for solving all problems in NP. In Practical Aspects of Declarative Languages, pp. 16-30.

Chai, D., \& Kuehlmann, A. (2003). A fast pseudo-boolean constraint solver. In Design Automation Conference, pp. 830-835.

Chaitin, G. J., Auslander, M., Chandra, A., Cocke, J., Hopkins, M., \& Markstein, P. (1981). Register allocation via coloring. Computer Languages, 6, 47-57.

Chams, M., Hertz, A., \& Werra, D. D. (1987). Some experiments with simulated annealing for coloring graphs. European Journal of Operations Research, 32, 260-266.

Coudert, O. (1997). Coloring of real-life graphs is easy. In Design Automation Conference, pp. $121-126$.

Crawford, J. (1992). A theoretical analysis of reasoning by symmetry in first-order logic. In AAAI Workshop on Tractable Reasoning at the Tenth National Conference on Artificial Intelligence.

Crawford, J., Ginsberg, M., Luks, E., \& Roy, A. (1996). Symmetry-breaking predicates for search problems. In 5th International Conference on Principles of Knowledge Representation and Reasoning, pp. 148-159.

Culberson, J. (2004). Graph coloring page. http://web.cs.ualberta.ca/ joe/Coloring/index.html.

Darga, P. T., Liffiton, M. H., Sakallah, K. A., \& Markov, I. L. (2004). Exploiting structure in symmetry generation for cnf. In 41 st Internation Design Automation Conference, pp. 530-534.

Davis, M., Logemann, G., \& Loveland, D. (1962). A machine program for theorem proving. Communications of the ACM, 5, 394-397. 
Fahle, T., Schamberger, S., \& Sellmann, M. (2001). Symmetry breaking. In 7th International Conference on Principles and Practice of Constraint Programming, pp. 93-107.

Feige, U., Goldwasser, S., Lovasz, L., Safra, S., \& Szege, M. (1991). Approximating clique is almost NP-complete. In IEEE Symposium on Foundations of Computer Science, pp. $2-12$.

Focacci, F., \& Milano, M. (2001). Global cut framework for removing symmetries. In Principles and Practice of Constraints Programming, pp. 77-82.

Galinier, P., \& Hao, J. (1999). Hybrid evolutionary algorithms for graph coloring. Journal of Combinatorial Optimization, 3, 379-397.

Garey, M. R., \& Johnson, D. S. (1979). Computers and Intractability: A Guide to the Theory of NP-completeness. W. H. Freeman and Company.

Gent, I. P. (2001). A symmetry-breaking constraint for indistinguishable values. In Workshop on Symmetry in Constraint Satisfaction Problems.

Goldberg, E., \& Novikov, Y. (2002). Berkmin: A fast and robust SAT-solver. In Design Automation and Test in Europe, pp. 142-149.

Haldorsson, M. M. (1990). A still better performance guarantee for approximate graph coloring..

Hentenryck, P. V., Agren, M., Flener, P., \& Pearson, J. (2003). Tractable symmetry breaking for CSPs with interchangeable values. In The International Joint Conference on Artificial Intelligence (IJCAI).

Hertz, A., \& Werra, D. D. (1987). Using tabu search techniques for graph coloring. Computing, 39, 345-351.

Huang, J., \& Darwiche, A. (2003). A structure-based variable ordering heuristic for SAT. In The International Joint Conference on Artificial Intelligence, pp. 1167-1172.

ILOG (2000). ILOG CPLEX ILP solver, version 7.0. http://www.ilog.com/products/cplex/.

J.-P. Hamiez, J.-K. H. (2001). Scatter search for graph coloring. In The 5th European Conference on Artificial Evolution, pp. 168-179.

Jagota, A. (1996). An adaptive, multiple restarts neural network algorithm for graph coloring. European Journal of Operational Research, 93, 257-270.

Kirovski, D., \& Potkonjak, M. (1998). Efficient coloring of a large spectrum of graph. In Design Automation Conference.

Krishnamurthy, B. (1985). Short proofs for tricky formulas. Acta Informatica, 22, 327-337.

Kubale, M., \& Jackowski, B. (1985). A generalized implicit enumeration algorithm for graph coloring. Communications of the ACM, 28, 412-418.

Kubale, M., \& Kusz, E. (1983). Computational experience with implicit enumeration algorithms for graph coloring. In Proceedings of the WG'83 International Workshop on Graph Theoretic Concepts in Computer Science, pp. 167-176.

Leighton, F. (1979). A graph coloring algorithm for large scheduling problems. Journal of Research of the National Bureau of Standards, 84, 489-506. 
McKay, B. D. (1990). Nauty user's guide (version 1.5). http://cs.anu.edu.au/^ bdm/nauty/.

Mehrotra, A., \& Trick, M. A. (1996). A column generation approach for graph coloring. INFORMS Journal on Computing, 8, 344-354.

Moskewicz, M., Madigan, C., Zhao, Y., Zhang, L., \& Malik, S. (2001). Chaff: Engineering an efficient sat solver. In Design Automation Conference, pp. 530-535.

Mycielski, J. (1955). Sur le coloriage des graphs. Colloqium Mathematicum, 3, 161-162.

Prestwich, S. (2002). Supersymmetric modelling for local search. In SymCon: Workshop on Symmetries in CSPs, pp. 21-28.

Puget, J. (2002). Symmetry breaking revisited. In Principles and Practice of Constraints Programming, pp. 446-461.

Ramani, A., Aloul, F. A., Markov, I. L., \& Sakallah, K. A. (2004). Breaking instanceindependent symmetries in exact graph coloring. In Design Automation and Test in Europe, pp. 324-329.

Roney-Dougal, C. M., Gent, I. P., Kelsey, T., \& Linton, S. (2004). Tractable symmetry breaking using restricted search trees. In European Conference on Artificial Intelligence, pp. 211-215.

Sheini, H. (2004). Pueblo 0-1 ILP solver. http://www.eecs.umich.edu/ ^hsheini/pueblo/.

Silva, J. P. M., \& Sakallah, K. A. (1999). GRASP: A new search algorithm for satisfiability. IEEE Transactions On Computers, 48, 506-521.

Trick, M. (1996). Network resources for coloring a graph. http://mat.gsia.cmu.edu/COLOR/color.html.

Walsh, T. (2001). Search on high degree graphs. In 17th International Joint Conference on Artificial Intelligence, pp. 266-271.

Warners, J. P. (1998). A linear-time transformation of linear inequalities into conjunctive normal form. Information Processing Letters, 68, 63-69.

Welsh, D. J. A., \& Powell, M. B. (1967). An upper bound on the chromatic number of a graph and its application to timetabling problems. Computer Journal, 10, 85-86.

Werra, D. D. (1985). An introduction to timetabling. European Journal of Operations Research, 19, 151-162. 